\title{
LncRNA-ZFAS1 Induces Epithelial-Mesenchymal Transition of Pancreatic Cancer Cells During Nutrient Deprivation by Promoting AMPK-Mediated Phosphorylation and Stabilization Of ZEB1 Protein
}

\author{
ZHU ZENG \\ Wuhan Union Hospital \\ Shengbo Han \\ Wuhan Union Hospital \\ Yang Wang \\ Wuhan Union Hospital \\ Yan Huang \\ Wuhan Union Hospital \\ Yuhang $\mathrm{Hu}$ \\ Wuhan Union Hospital \\ Fengyu Xu \\ Wuhan Union Hospital \\ Yong Zhao \\ Wuhan Union Hospital \\ Mengqi Huang \\ Wuhan Union Hospital \\ Jiang Tang \\ Wuhan Union Hospital \\ Decai Wang \\ Wuhan Union Hospital \\ Gang Zhao ( $\nabla$ gangzhao@hust.edu.cn ) \\ Wuhan Union Hospital
}

Research

Keywords: Pancreatic cancer, IncRNA, ZFAS1, ZEB1, EMT, metastasis

Posted Date: September 16th, 2021

DOI: https://doi.org/10.21203/rs.3.rs-875697/v1 
License: (c) (i) This work is licensed under a Creative Commons Attribution 4.0 International License. Read Full License 


\section{Abstract}

Background: Nutrient deprivation is a distinct feature of the tumor microenvironment that plays a crucial role in various cancers. However, the contribution and regulatory mechanism of nutrient deprivation on metastasis of pancreatic cancer (PC) have not been identified.

Methods: PC cells were treated with normal medium, glucose-depletion or glutamine-depletion medium to observe the epithelial-mesenchymal transition (EMT). RT-qPCR and western blot assay were applied to evaluate the alteration of mRNA and protein of zinc finger E-box binding homeobox 1 (ZEB1), a crucial EMT regulator factor. Co-IP assay was utilized for evaluating the interaction between AMP-activated protein kinase (AMPK) and ZEB1. LncRNA microarray was adopted to detect the potential IncRNA, which facilitates the association between AMPK and ZEB1. Gain- and loss-of-function experiments were performed to evaluate the roles of ZNFX1 antisense RNA 1 (ZFAS1) in EMT and metastasis of PC.

Results: The present study reveals that nutrient deprivation including glucose and glutamine deprivation significantly induces EMT of PC cells, which is dependent on stabilization of ZEB1. We further discover that nutrient deprivation induces upregulation of IncRNA ZFAS1, which promotes the association between AMPK and ZEB1 to phosphorylate and stabilize ZEB1 protein. Notably, ZEB1 reciprocally promotes the transcription of ZFAS1 by binding to the promoter of ZFAS1, forming feedback with ZFAS1. Consistently, depletion of ZFAS1 obviously inhibits nutrient deprivation-induced EMT of PC cells and lung metastasis of PC in nude mice. Meanwhile, clinical data displays that ZFAS1 is overexpressed in PC tissues and correlated with high expression of ZEB1 and Vimentin (VIM), low expression of E-cadherin (E-cad), as well as poor prognosis in $\mathrm{PC}$ patients.

Conclusions: Our study implicates that glucose and glutamine deprivation promotes EMT and metastasis of PC through IncRNA-mediated stabilization of ZEB1.

\section{Background}

Pancreatic ductal adenocarcinoma (PDAC), one of the most aggressive malignant tumors, is expected to be the second most common cause of cancer-related deaths by 2030 with a 5-year overall survival rate of $9 \%[1-3]$. Inaccessible location of the pancreas, unspecific symptoms and lack of reliable biomarkers have hampered the diagnosis [4]. Consequently, most pancreatic cancer (PC) patients are diagnosed as being frequently accompanied by metastatic lesions. Only less than $20 \%$ of patients can undergo surgical resection, and most patients must undergo systemic treatment. Although neoadjuvant chemotherapy has achieved effects, they can only prolong the life of patients by 6-12 months $[5,6]$. Hence, actively exploring early detection methods and more effective prevention strategies is of great significance to improve the survival rate of PDAC patients.

The tumor microenvironment (TME) is an extremely complex comprehensive system composed of tumor cells, extracellular matrix components and non-tumor cells [7]. Due to the solid pressure and fluid pressure generated in the tumor and the compression of the surrounding blood vessels, this dense tumor 
mass forms a highly hypoxic and malnourished microenvironment [8-10]. More importantly, a metabolomics study used mass spectrometry to compare samples of PDAC patients with nearby benign tissues and found that poor tumor perfusion contributed to not only a large depletion of oxygen, but also nutrients, including glucose, glutamine and fatty acids [11], indicating that PDAC cancer cells must survive by displaying unconventional and modified pathways in order to adapt to the pressure brought by the microenvironment.

Epithelial-mesenchymal transition (EMT) is a biological process in which epithelial cells are transformed into cells with mesenchymal phenotype and plays a critical role in inducing increased infiltration and distant metastasis of many types of tumors, as well as the generation and maintenance of cancer stem cells $[12,13]$. Increasing evidence indicates that nutrient stress induced by limited oxygen, glucose and glutamine contributes to EMT of cancer cells. Hypoxia has been recognized to have a central role in the induction and development of EMT mainly through hypoxia/HIF-dependent regulation in PDAC [14, 15]. Additionally, glucose deprivation was demonstrated to have a critical role in mediating EMT under hypoxia in hepatic cancer cells [16]. Furthermore, a study clarified that Slug was attributed to EMT, cell motility, and nutrient stress survival induced by glutamine deprivation in PDAC [17]. Nevertheless, little is known about the regulatory mechanism of EMT in pancreatic driven by glucose and glutamine deprivation.

Long non-coding RNAs (IncRNAs) are non-protein coding RNA transcripts longer than 200 nucleotides that emerge as critical regulators in various cellular processes [18]. Increasing evidence shows that IncRNA is involved in the remodeling of tumor cells under nutritional stress. In our previous study, we ascertained that antisense RNA of glutaminase (GLS-AS) was downregulated in PDAC cells under glutamine depletion, which reduced the inhibition of GLS expression, thereby promoting proliferation and migration of PC cells [19]. Another study showed that AMP-activated protein kinase (AMPK) pathway induced IncRNA-NBR2 overexpression under energy stress in kidney cancer and breast cancer cells, which in turn activated the activity of AMPK kinase and inhibited cell proliferation [20]. Meanwhile, results from Chen et al showed that IncRNA-TP53TG1 promoted glioma cell proliferation and migration by regulating expression of glucose metabolism related genes under glucose deprivation [21]. Therefore, we sought out to figure out whether specific IncRNA contributes to the adaptive EMT of PC under glucose and glutamine deprivation.

In our present study, we delineated that PC cells displayed EMT with enhanced capacity of migration and invasion under glucose and glutamine deprivation. Results further revealed that IncRNA- ZNFX1 Antisense RNA 1 (ZFAS1) was the pivotal regulator for EMT during nutrient stress by stabilizing ZEB1 protein via AMPK-mediated phosphorylation. Moreover, the increase of ZFAS1 under nutrient stress was ascribed to transcriptional regulation of ZEB1, which formed a reciprocal feedback between ZFAS1 and ZEB1.

\section{Methods}


Patients and tumor specimens

PC and corresponding peri-tumor tissues of 60 surgical resections of patients without preoperative treatment were provided by the Institute of Pancreatic Diseases of Union Hospital (Wuhan, China) from 2013 to 2017. The Ethics Committee of the Academic Medical Center of Huazhong University of Science and Technology approved the procedure of human specimen collection (Permission No: 2013, S199), and all patients signed informed consent. The tissues were delivered to the laboratory within $20 \mathrm{~min}$, and then either formalin fixed and paraffin-embedded or snap frozen as described previously [22].

Cell culture

PANC-1 and BxPC-3 cell lines were originally obtained from the ATCC (American Type Culture Collection). All cell lines were subjected to authentication using short tandem repeat multi-amplification and tested negative for mycoplasma contamination. PANC-1 cells were grown in DMEM (Gibco, USA) medium. BxPC-3 cells were grown in RPMI-1640 (Gibco, USA). All the medium contained $10 \%$ fetal bovine serum (FBS, Gibco), $100 \mathrm{U} / \mathrm{mL}$ penicillin, and $100 \mathrm{mg} / \mathrm{mL}$ streptomycin. For glucose or glutamine deprivation, cells were plated overnight in complete DMEM, briefly washed with phosphate-buffered saline (PBS) and then transferred into glucose-free DMEM (Thermo Fisher, catalogue \#11966025) or glutamine-free RPMI 1640 (Thermo Fisher, catalogue \#21870076) supplemented with 10\% dialyzed FBS (Gibco, catalogue \#26400), respectively.

\section{Cell-proliferation assay}

Firstly, the cultured cells were digested with $0.4 \%$ trypsin and counted. Plant the cells in 96-well plate with 2000 cells per well, and set a blank control well for a total of 5 plates. From the next day, take out a 96well plate at the same time every day. Add $20 \mu$ of 3-(4,5-dimethylthiazol-2-yl)-2,5-diphenyltetrazolium bromide (MTT, Sigma, $5 \mathrm{mg} / \mathrm{ml}$ ) to the well. Place the plate in an incubator at $37^{\circ} \mathrm{C}$ in the dark for $4 \mathrm{~h}$. Then replace the medium with $150 \mu \mathrm{LMSO}$ (Sigma). After the cells were completely lysed, the viability of cells was measured by measuring the absorbance at $570 \mathrm{~nm}$ using an ELISA reader (Thermo Fisher Scientific). The experiment above was repeated 3 times independently.

Transwell and wound healing assay

Cell invasion and migration were evaluated using Transwell inserts (Corning-Costar; pore size $8 \mu \mathrm{m}$ ) with or without Matrigel (Sigma) coating. Cells were resuspended in FBS-free media prior to addition to the upper chamber of wells containing this insert, with media containing $30 \%$ FBS added to the lower portion. Cells were then incubated for $24 \mathrm{~h}$, after which those not invading were removed, and the remaining cells under the membrane were fixed and stained. The number of invasive and migrated cells in 5 random fields was then determined via light microscopy. Additionally, the wound healing experiment was utilized to measure the migration ability of cells. The cells were plated until they were confluent in the wells of the 6-well plate, and then a sterile pipette tip was employed to scrape the monolayer of cells. Cell images at 0 $\mathrm{h}$ and $24 \mathrm{~h}$ post-wounding were used to gauge migration rates. 
Reverse transcription quantitative polymerase chain reaction (RT-qPCR)

TRIzolß Reagent (Invitrogen) was purposed to extract total RNA from PC cells and tissues. PrimeScript RT Master Mix (RR036A, Takara) was utilized for the RNA reverse-transcribed into cDNA according to provided directions. Real-time quantitative PCR was performed using TB Green ${ }^{B}$ Premix Ex Taq ${ }^{\mathrm{TM}}$ (RR420A, Takara) based on the manufacturer's recommendations. $\beta$-actin acted as a control for cellular RNA. The primers sequences used in this research were placed in Table S1.

\section{Western Blot}

Ice-cold PBS was employed to wash the cells 2-3 times. After adding RIPA lysis buffer supplemented with protease inhibitor Cocktail (1:50) and phenylmethyl sulfonyl fluoride (PMSF, 1:100), protein was extracted immediately. An appropriate amount of 5xsodium dodecyl sulfate-polyacrylamide gel electrophoresis (SDS-PAGE) protein loading buffer was added into the protein sample in a 4:1 ratio. Boil water for $10 \mathrm{~min}$ to denaturant and store at $-2^{\circ} \mathrm{C}$. Protein was separated using SDS-PAGE gel, subsequently transferred onto polyvinylidene fluoride membranes (PVDF, Millipore, USA). Membranes were blocked in $5 \%$ non-fat milk for $1 \mathrm{~h}$ at room temperature. Then incubated with specific primary antibodies overnight at $4^{\circ} \mathrm{C}$ followed by the incubation of anti-mouse or rabbit horseradish peroxidase (HRP)-conjugated secondary antibodies (\#7076S/7074S, CST, dilution 1:3000). Afterwards, the enhanced chemiluminescence assay (ECL, 34095, Pierce) was implemented to visualize the band signals, collected by the ChemiDoc XRS molecular imager system (Bio-Rad, USA). Primary antibodies against ZEB1 (215441-AP, Proteintech, dilution 1:1000), AMPK (\#2757, CST, dilution 1:1000), Phospho-AMPKa (Thr172) (\#50081, CST, dilution 1:1000), Phospho-AMPK Substrate Motif (\#5759, CST, dilution 1:1000), Ubiquitin (10201-2-AP, Proteintech, dilution 1:1000), PAN-AC (66289-1-lg, Proteintech, dilution 1:1000), PhosphoSer/Thr (ab117253, Abcam, dilution 1:1000), E-cad (\#3195, CST, dilution 1:1000), VIM (\#5741, CST, dilution 1:1000) and $\beta$-actin (20536-1-AP, Proteintech, dilution 1:1000) were used.

\section{Co-immunoprecipitation (Co-IP)}

The Co-IP assay for AMPK and ZEB1 was conducted via lysing PANC-1 or BxPC-3 cells with Pierce IP lysis buffer (\#87787, Thermo) containing protease inhibitor cocktails and PMSF on ice for 30 min followed by sonication (power 10 for three cycles, $10 \mathrm{~s}$ on and $50 \mathrm{~s}$ for rest) to release nuclear proteins completely. One tenth of the lysate was extracted as input. The remaining lysate was then incubated with IgG, ZEB1 or AMPK antibody overnight at $4^{\circ} \mathrm{C}$ followed by the incubation of Protein A/G Magnetic Beads (Bimake, Houston, TX, USA) at $4^{\circ} \mathrm{C}$ for $2 \mathrm{~h}$. Then the protein/agarose complex was isolated and resuspended in loading buffer, degenerated and further analyzed by Western Blot.

\section{Microarray analysis}

The method is similar to the previous article [22]. KangChen Bio-tech (Shanghai, China) provided the Arraystar human LncRNA microarrays V3.0. Total RNA was collected from PANC-1 cells cultured with complete medium, glucose-free medium or glutamine-free medium using TRIzolß Reagent (Invitrogen). 
NanoDrop ND-1000 was employed to evaluate the RNA quantity and purity. Standard denaturing agarose gel electrophoresis was conducted to assess RNA integrity. Quick Amp Labeling Kit, One-Color (Agilent), was utilized to amplify RNA and label to generate cDNA. The cDNA samples were purified and labeled with a Low Input Quick-Amp Labeling Kit (Agilent) according to the manufacturer's instructions. After further purification, the cDNA samples were microarray hybridized at $65^{\circ} \mathrm{C}$ for $17 \mathrm{~h}$ according to the instructions of the gene expression hybridization kit (Agilent). Then a microarray scanner (Agilent) was used to scan all microarrays.

RNA immunoprecipitation (RIP)

Magna RIP ${ }^{\text {TM }}$ RNA-Binding Protein Immunoprecipitation Kit (Millipore, USA) was applied to perform the RIP assay according to the provided direction. Cells were lysed by the Pierce IP lysis buffer (\#87787, Thermo), blended with protease inhibitor (cocktails/PMSF) and RNase inhibitors. One tenth of the lysate was extracted as input. Subsequently, Magnetic beads were pre-incubated with IgG, ZEB1 or AMPKa2 antibody separately for $30 \mathrm{~min}$ at room temperature. Then mixed the washed Magnetic bead antibody complex and the remaining cell lysates at $4^{\circ} \mathrm{C}$ overnight. The co-precipitated RNA was purified using TRIzol® Reagent (Invitrogen) according to the manufacturer's protocol. After RT-qPCR, 2\% agarose gel electrophoresis was performed to detect the precipitated RNA.

RNA pulldown

RNA pulldown assay was performed as previously described [22]. The double-stranded DNA template was amplified by RT-qPCR. Then the ZFAS1 and antisense of ZFAS1 were transcribed using the MAXIscript ${ }^{\circledR}$ Kit (Ambion, USA) with T7 phage RNA polymerases employed to mix together with RNA polymerase, rNTPs, and transcriptional buffer for in vitro transcription with double-stranded DNA as the template. The Pierce ${ }^{\text {TM }}$ RNA 3' End Desthiobiotinylation Kit (Thermo Scientific, USA) was utilized to attach biotinylated nucleotide to the 3 'terminal of ZFAS1 and antisense of ZFAS1. The Pierce ${ }^{\text {TM }}$ Magnetic RNAProtein Pulldown Kit (Thermo Scientific, USA) and MAXIscript ${ }^{\circledR}$ Kit (Ambion, USA) were used for RNA pulldown. Prepare cell lysate and extract one-tenth of it as input. The remaining cell lysates were incubated with complex of biotinylation labeled RNA and magnetic beads. After elusion, samples were analyzed using Western Blot. All the procedures were conducted as the given instructions.

Transfection

Transfection of specific siRNAs for ZEB1, AMPK, ZFAS1 or negative control were purchased from GenePharma (Shanghai, China). All the overexpression plasmids of full-length ZEB1, ZFAS1 and truncations of ZFAS1 and empty vector purchased from GeneChem (Shanghai, China). For stable transfection, full-length ZEB1 and ZFAS1 siRNA were constructed into lentiviral vector, with empty vector as a negative control. Lipofectamine 2000 (Invitrogen, USA) was used for cell transfection according to the manufacturer's protocol. The sequences of overexpression plasmids and siRNAs were placed in Table S2. 
Chromatin immunoprecipitation (ChIP)

ChIP assay was performed in the directions of the ChIP assay kit (Millipore, Lot \# 2983461, USA). Enough cells were first cross-linked with $1 \%$ formaldehyde for $10 \mathrm{~min}$, and then the cells were lysed according to the protocol in the kit. All the procedures were conducted on ice. Ultrasonic treatment was employed to shear the DNA. ZEB1 or IgG antibodies and agarose beads were added into the lysate for immunoprecipitation reaction. The precipitated complex of protein and DNA was then eluted and purified. The isolated DNA fragments were subjected to PCR, and the PCR products were analyzed by $2 \%$ agarose gel electrophoresis.

\section{Luciferase reporter assay}

PANC-1/BxPC-3 cells were co-transfected with pRL-TK plasmid encoding Renilla luciferase and luciferase reporter plasmid encoding firefly luciferase or/and ZEB1/ZFAS1 promoter sequence (wild-type or mutanttype) for $24 \mathrm{~h}$. Then the cells were cultured under glucose and glutamine depletion for $24 \mathrm{~h}$. The luciferase activities were evaluated using a luciferase activity kit (Promega, USA). Renilla luciferase served as a normalizing control. The procedure was summarized as follows: the cells were digested with 0.4\% trypsin and seeded into 96-well plates. On the second day, the cells were lysed using a diluted Passive Lysis Buffer. Then add the Luciferase Assay Buffer II to measure the firefly luciferase activity. Add 1XStop\&Glo® Reagent to stop the reaction and measure the second fluorescence value, the Renilla luciferase. Promoter activity per well = firefly luciferase activity/Renilla luciferase activity. The experiment above was repeated 3 times independently.

Immunohistochemistry (IHC)

The paraffin-embedded tissues were sectioned and dewaxed with xylene. Then wash with different concentrations of alcohol (100\%, 95\%, 80\%, 70\%) and distilled water respectively. Soaked in 3\% hydrogen peroxide for $10 \mathrm{~min}$, followed by washing with distilled water. Added citric acid buffer $\mathrm{pH}=6$ and cook in microwave for 10 min. After cooling to room temperature, wash with distilled water and PBS, add 10\% goat serum drop by drop, and place it in the $37^{\circ} \mathrm{C}$ incubator for $30 \mathrm{~min}$. After incubating with anti-ZEB1, ECad, VIM primary antibodies and biotin secondary antibodies, DAB solution was added on the tissues. Then hematoxylin counterstained, dehydrated, transparent, and sealed were performed. Finally, the inverted microscope was utilized to collect the image of the stained section.

\section{Xenograft assay}

BALB/c male nude mice (4 weeks old) were purchased from HFK Bio-Technology Co (Beijing, China). The mice were randomly divided into four groups $(n=6)$. PANC- 1 cells $\left(2 \times 10^{6} / \mathrm{mice}\right)$ transfected with LVControl, LV-ZEB1 or LV-siZFAS1 were subcutaneously injected into nude mice from the tail vein. The growth of mice was observed every 1-3 days. The date of death was recorded. Visible metastases on lung were counted and displayed by hematoxylin and eosin (H\&E)-stained slides. The Institutional 
Animal Care and Use Committee of Tongji Medical College, Huazhong University of Science and Technology (Wuhan, China) approved the study.

\section{Statistical analysis}

All experiments were conducted at least 3 times. SPSS 22.0 (IBM, USA) and GraphPad Prism 8 (GraphPad Software, USA) were utilized for all the statistical analysis. The results are presented as the mean \pm S.D. analyzed by Student's $t$ test or one-way ANOVA. Relative gene expression was analyzed using the $2^{-\Delta \Delta C t}$ method. Survival curves were performed using the Kaplan-Meier method and assessed using the logrank test. All the statistical tests were two-sided. The difference was considered to be significant at *, $\mathrm{P}<$ $0.05, * *, P<0.01, * \star *, P<0.001$. NS, no significant.

\section{Results}

\section{EMT is an adaption of PC cells in response to glucose and glutamine deprivation}

To investigate the adaption of PC cells in response to nutrient stress, we cultured human PC cells under saturating nutrient medium or nutrient deprivation medium with glucose-free or glutamine-free up to $72 \mathrm{~h}$. With the extension of culture time, cells incubated in medium lacking glucose and glutamine undergo dramatically EMT-like transformation, evidenced by alterations of morphology from an epithelial (a polygonal shape and cobblestone-like sheets) to a more mesenchymal (loose cell contacts, scattered from cell cluster and acquired an elongated, fusiform morphology with dendritic processes) phenotype (Fig. 1a). Compared to cells grown in nutrient medium, cells cultured under glucose and glutamine depleted medium displayed with retarded proliferation (Fig. 1b). Meanwhile, results of transwell and wound healing assay indicated that the invasion and migration of PC cells were remarkably enhanced by glucose and glutamine depletion (Fig. 1c-e). Moreover, Western Blot assay demonstrated elevation of ZEB1 and vimentin (VIM) protein and decrease of E-cadherin (E-cad) protein in the PC cells under nutrient depletion (Fig. 1f). Of note, different from the mRNA of VIM and E-cad, RT-qPCR results displayed no obvious alteration of ZEB1 mRNA (Fig. 1g), which indicated a post-transcriptional regulation of ZEB1 expression during nutrient starvation. Collectively, these data revealed that EMT would be an adaptive response of PC cells during glucose and glutamine deprivation.

\section{Upregulated ZEB1 protein is a critical regulator for nutrient starve-induced EMT of PC cells}

As a driver of EMT, ZEB1 inhibits or activates expression of E-cad and VIM via binding to the promoters of E-cad and VIM as a transcription factor $[23,24]$. To determine the functional significance of upregulated ZEB1 protein in PC cells suffering nutrient stress, ZEB1 was depleted by siRNAs in PANC-1 and BxPC-3 cells. It was observed that ZEB1 depletion significantly decreased expression of VIM and increased expression of E-cad, with reversal morphological changes of EMT in PC cells, which was induced by glucose and glutamine depletion (Fig. 2a and b). Moreover, ZEB1 inhibition could inhibit the increased ability of invasion and migration in PC cells in response to glucose nor glutamine starvation (Fig. 2c and 
d, Additional file 1: Fig. S1A). Thus, the results implied that upregulated ZEB1 was decisive for the nutrient deprivation-induced EMT of PC cells.

\section{ZEB1 protein was regulated at post-translational level in response to nutrient depletion via phosphorylation modification}

Then we ought to elucidate the underlying potential mechanisms by which ZEB1 protein was regulated during nutrient deprivation. To further validate the upregulation of ZEB1 was due to post-transcriptional regulation, luciferase plasmid containing the promoter region of ZEB1 was constructed to evaluate the activation of ZEB1 promoter under glucose or glutamine deprivation. The results indicated that the promoter activity of ZEB1 had no visible change during glucose and glutamine depletion (Fig. 3a). Meanwhile, the protein synthesis inhibitor cyclohexane (CHX) was employed to detect the protein stability of ZEB1. The results showed that exposure under the glucose and glutamine starvation remarkably enhanced the half-life of ZEB1 protein in both PANC-1 and BXPC-3 cells (Fig. 3b). Moreover, the nutrient depletion-induced ZEB1 expression could be abolished by the proteasome inhibitor MG132 (Fig. 3c). These results further implied that ZEB1 might be regulated at post-translational level under nutrient stress. To further identify the post-translational regulation of ZEB1, the common protein modifications including acetylation, ubiquitination, and phosphorylation were evaluated in PC cells, respectively. Interestingly, the acetylation and ubiquitination of ZEB1 showed no significant changes in PC cells with glucose and glutamine starvation, while phosphorylation of ZEB1 was profoundly increased (Fig. 3d-f). Collectively, ZEB1 protein was regulated at post-translational level via phosphorylation under glucose and glutamine starvation.

\section{AMPK-dependent phosphorylation at Thr220 mediates the stabilization of ZEB1 under nutrient starvation}

AMPK is a crucial cellular energy sensor, which was activated once exposure to the microenvironment of low nutrients and oxygen, leading to phosphorylation of a number of key players in regulating the tumor or anti-tumor process $[25,26]$. AMPK substrates have a common phospho-AMPK substrate motif [LXRXX(pS/pT)] [27]. Analysis of the ZEB1 protein sequence revealed an evolutionarily conserved phospho-AMPK substrate motif encompassing 215-LERHMT-220 (Fig. 4a). Therefore, we presumed whether ZEB1 was regulated by AMPK via phosphorylation. Consistently, the Co-IP assay revealed the physical interaction between ZEB1 and AMPK (Fig. 4b). Moreover, the Co-IP assay validated ZEB1 would be a phosphorylated substrate of AMPK via utilizing the phospho-AMPK (p-AMPK) substrate motif antibody (LXRXX (pS/pT), referring to pS/pT) (Fig. 4c). Additionally, depletion of AMPK with siRNA significantly downregulated ZEB1 protein levels with no changes in mRNA level (Fig. 4d, e). Consistently, both knockdown AMPK and treatment with the AMPK inhibitor, Compound C [28] (refer to C.C), successfully eliminated the phosphorylation and expression of ZEB1, as well as the interaction between ZEB1 and p-AMPK which were enhanced during nutrient deprivation (Fig. 4f, g). To further validate ZEB1 is stabilized by AMPK through phosphorylating at Thr220, the Flag-tagged wild type (WT) and mutant type (MUT, threonine to alanine) of ZEB1 plasmids were transfected into PANC-1 cells. The Western Blot results revealed that threonine-to-alanine replacement at Thr220 abolished the phosphorylation and 
expression of flag-ZEB1, as well as the interaction between p-AMPK and flag-ZEB1 upon glucose and glutamine starvation (Fig. 4h). Taken together, the data above revealed that the upregulation of ZEB1 protein was attributed to AMPK-mediated phosphorylation and stabilization.

\section{ZFAS1 facilitates interaction between AMPK and ZEB1}

Recent literature has elucidated that IncRNAs participated in regulating cellular metabolism [18, 19]. Meanwhile, IncRNA has been defined to serve as molecular scaffolds to connect different proteins that need to play a synergistic role [29]. Therefore, it drew our interest in whether IncRNA was involved in the interaction of AMPK and ZEB1 during nutrient deprivation. Notably, the combination between AMPK and ZEB1 was substantially decreased when samples were pretreated with RNase (Fig. 5a), indicating a possibility that IncRNA affecting the binding between AMPK and ZEB1. To identify the potential IncRNAs, we conducted IncRNA microarray in PANC-1 cells cultured with complete medium, glucose-free medium or glutamine-free medium (Fig. 5b). The catRAPID and RNA-Protein Interaction Prediction (RPISeq) database [30,31] were utilized to predict the IncRNA that may bind to AMPK and ZEB1, respectively. By intersecting analysis of microarray and database, ZFAS1 was screened as AMPK-ZEB1 binding IncRNA, and upregulated under glucose-free and glutamine-free conditions (Fig. 5c). RT-qPCR assay further validated that ZFAS1 was remarkably elevated in PANC-1/BxPC-3 cells under glucose and glutamine starvation (Fig. 5d). To determine whether ZFAS1 specifically binds to ZEB1 and AMPK, RNA pulldown and RIP assays were performed. The results showed that ZFAS1 but not antisense of ZFAS1 bind to AMPK and ZEB1 (Fig. 5e and f, Additional file 1: Fig. S2A, B). Domain truncation constructs of ZFAS1 were employed to clarify the structural basis for these interactions. It is interesting to note that the 1250nt of ZFAS1 was observed to bind to both ZEB1 and AMPK (Additional file 1: Fig. S2C). Consistently, the amount of co-associated p-AMPK within ZEB1 immunoprecipitation and ZEB1 protein level were substantially reduced when ZFAS1 was silenced (Additional file 1: Fig. S2D and E). Conversely, ZFAS1 overexpression significantly enhanced the combination between AMPK and ZEB1, as well as ZEB1 expression (Additional file 1: Fig. S2F and G). Additionally, the mRNA level of ZEB1 had no change in ZFAS1 knockdown or overexpression PC cells. In support, the amount of co-associated AMPK within ZEB1 immunoprecipitation and ZEB1 protein level were elevated in PANC-1 cells transfected with ZFAS11 overexpression plasmid which encoding the 250nt of ZFAS1, but not in that of ZFAS1-dt1 plasmid with the 250nt of ZFAS1 deletion (Additional file 1: Fig. S2H). Additionally, ZFAS1 knockdown eliminated the ZEB1 expression and associated p-AMPK protein level induced by glucose and glutamine starvation (Fig. $5 \mathrm{~g}$ ). Collectively, the findings suggest that ZFAS1 drives the association between p-AMPK and ZEB1.

\section{ZFAS1 enhances ZEB1 protein stability through promoting the AMPK-mediated phosphorylation}

To further investigate the mechanism that ZFAS1 regulates ZEB1 expression, we detected the stability of ZEB1 protein in ZFAS1 knockdown PC cells under glucose and glutamine starvation. The results indicated that the ZFAS1 silencing distinctively decreased stability of ZEB1 protein in glucose and glutamine-depleted PANC-1 and BXPC-3 cells (Fig. 6a, b). Results of Co-IP assay showed that the AMPK activator, A769662 and AICAR, significantly promoted phosphorylation of ZEB1, but consequently 
inhibited by knockdown of ZFAS1 (Fig. 6c, d). Meanwhile, phosphorylation of ZEB1 was increased during nutrient starvation, which could be decreased by AMPK inhibitor, compound $C$ and further rescued by overexpression of ZFAS1 (Fig. 6e, f). Taken together, these findings suggest that ZFAS1 facilitates the AMPK-mediated phosphorylation and stabilization of ZEB1 under glucose and glutamine depletion.

\section{ZFAS1 is transcriptionally regulated by ZEB1 under glucose and glutamine deprivation}

Next, we sought to elucidate the mechanism of ZFAS1 upregulation in PC cells under glucose and glutamine starvation. Luciferase reporter was designed to test the transcriptional activity of ZFAS1. The result indicated that the transcriptional activity of ZFAS1 was significantly enhanced in glucose and glutamine-depleted PC cells (Fig. 7a). Analysis of transcription factor binding profiles by Jaspar indicated

a ZEB1 potential binding site located on the promoter of ZFAS1 gene. In support, ChIP assay showed that ZFAS1 promoter sequence containing -152nt -CTCACCCGGCT- -162nt sequence was captured by ZEB1 immunoprecipitation, which was enhanced in glucose and glutamine starved PC cells (Fig. 7b). To prove that ZEB1 specifically binds to the ZFAS1 promoter, ZFAS1 promoter sequences carrying -152nt CTCACCCGGCT- -162nt sequence (wild type, WT) or mutant -152nt -CTAAAAAAAAA- -162nt sequence (mutant type, MUT) were inserted into a luciferase reporter vector and transfected into PC cells. The results showed that the promoter activity of ZFAS1 in WT cells, but not MUT cells, was notably enhanced during glucose and glutamine depletion, which could be reversed by ZEB1 knockdown (Fig. 7c, d). Coincidently, RT-qPCR assays further validated ZEB1 knockdown remarkably inhibited the increase of ZFAS1 upon glucose and glutamine depletion (Fig. 7e). Accordingly, AMPK activator, A769662 and AICAR, enhanced both the promoter activity and expression of ZFAS1, which was also abolished by ZEB1 knockdown (Fig. 7f, g). Collectively, these results verified a requirement of ZEB1 for the upregulation of ZFAS1 in PC cells under nutrient depletion, which formed a reciprocal feedback between ZEB1 and ZFAS1.

\section{ZFAS1/ZEB1 feedback is critical for the nutrient stress-induced EMT and metastasis of PC cells}

Next, we characterize the role of ZFAS1 in PC cells under glucose and glutamine starvation. The data showed that ZFAS1 knockdown distinctively inhibited the enhanced invasion and migration of PC cells in response to glucose and glutamine starvation (Fig. 8a-C, Additional file 1: Fig. S3A-C). Meanwhile, the Western Blot assay revealed that knockdown of ZFAS1 visibly decreased protein expression of ZEB1 and VIM, but increased that of E-cad which was reduced during nutrient deprivation (Fig. 8d, Additional file 1: Fig. S3D). In further study, we found that the athymic nude mice injected with PANC-1 cells with stable ZFAS1 overexpression exhibited increased lung metastatic colonies and shorter survival, which was remarkably reversed by ZEB1 knockdown (Fig.8e-g), indicating that ZFAS1/ZEB1 loop is critical for the metastasis of PC cells. Overall, these findings demonstrated that ZFAS1 was involved in the role of ZEB1 in promoting EMT and metastasis of PC cells under glucose and glutamine depletion.

\section{ZFAS1 is highly expressed in PC and correlated with poor prognosis}


To determine whether these findings above are clinically relevant, we compared the ZFAS1 expression in various cancer tissues. Analysis from database GEPIA displayed that ZFAS1 expression was upregulated in tumor tissues including pancreatic adenocarcinoma (PAAD), colon adenocarcinoma (COAD), Rectum adenocarcinoma (READ), and liver hepatocellular carcinoma (LIHC) (Fig. 9a). Meanwhile, the same trend was observed in Lymphoid Neoplasm Diffuse Large B-cell Lymphoma (DLBC), Thymoma (THYM), Glioblastoma multiforme (GBM), Brain Lower Grade Glioma (LGG), Uterine Carcinosarcoma (UCS), Kidney renal clear cell carcinoma (KIRC), and Testicular Germ Cell Tumors (TGCT) (Additional file 1: Fig. S4A). Consistently, the result of RT-qPCR assay indicated a stronger expression of ZFAS1 in 60 PC tissues, compared with adjacent normal tissues (Fig. 9b). Kaplan-Meier survival curve analysis (K-M analysis) indicated that higher ZFAS1 expression in PC patients was associated with shorter survival (Fig. 9c). We also found that the ZFAS1 was negatively correlated with mRNA level of E-cad expression, but positively correlated with that of VIM in PC tissues (Fig. 9d, e). Additionally, the IHC assay showed that ZFAS1 was positively correlated with protein level of ZEB1 and VIM, but negatively associated with protein expression of E-cad (Fig. 9f). Therefore, these results further support that ZFAS1 is an oncogenic IncRNA for PC.

Taken together, our results indicate that nutrient deprivation induces an AMPK-mediated feedback of ZFAS1 and ZEB1, which consequently promotes EMT and metastasis of PC. (Fig. 9g).

\section{Discussion}

Cancer cells are believed to deal with fluctuations and limit nutrient levels in an unstable microenvironment to ensure their vitality and reproduction. Glucose and glutamine are the crucial metabolites of catabolism and anabolism, which are the focus of many diagnostic methods and therapeutic targets [10]. Understanding the adaption of tumor cells during nutrients stress including glucose and glutamine depletion is of great significance to tumor treatment. In this study, we revealed that PC cells exhibited EMT process with increased invasion and migration abilities under nutrient stress caused by glucose or glutamine starvation. Our work elucidated a new mechanism that the feedback between IncRNA-ZFAS1 and ZEB1 was the hub regulator for the adaption of PC cells during nutrient deprivation.

Due to the rapid proliferation, abnormal proliferation of blood vessels and tumor heterogeneity, the tumor microenvironment in PC often presents a state of nutritional stress such as low glucose and low glutamine. Numerous studies have reported that glucose and glutamine deprivation could contribute to the EMT of cancer cells. It was clarified that glucose deprivation can promote EMT of liver cancer cells under hypoxic conditions [16]. Meanwhile, Recouvreux et al demonstrated that glutamine starvation promoted EMT and metastasis of PC cells via regulating Slug [17]. Being an evolutionarily conserved developmental program, EMT was demonstrated to confer metastatic properties via enhancing mobility and invasion of cancer cells [32]. Coincidently, our research also revealed that both glucose and glutamine deprivation remarkably induced EMT of PC cells, which provides further evidence that the tumor microenvironment exerts critical roles in tumorigenesis. 
Although the role of ZEB1 in EMT and metastasis has been widely reported [33], whether ZEB1 contributes to the metastasis and EMT during nutrient deprivation has not been revealed yet. In the present study, we found that ZEB1 was obviously upregulated in PC cells during nutrient deprivation, accompanied with upregulation of VIM and downregulation of E-cad. Meanwhile, after knockdown of ZEB1, both the metastasis and EMT of PC cells under nutrient deprivation were remarkably impeded. These results indicated that ZEB1 is a decisive regulator for adaption of PC cells during nutrient stress caused by glucose and glutamine starvation.

Then we ought to investigate the underlying mechanism of upregulation of ZEB1 protein during nutrient deprivation. Notably, our results showed that only the protein but not the mRNA of ZEB1 was remarkably increased, indicating a post-transcriptional regulation. Furthermore, we revealed the stabilization of ZEB1 was distinctively induced by nutrient deprivation. The post-translational modifications of proteins include phosphorylation, acetylation, ubiquitination, etc [34]. Study had elucidated that ZEB1 was phosphorylated and stabilized by the kinase ATM in response to DNA damage, which promoted tumor cell radioresistance in vitro and in vivo [35]. Similarly, our results showed that only phosphorylation but not ubiquitination and acetylation of ZEB1 was obviously increased under nutrient deprivation. Therefore, our results indicated that the nutrient deprivation-induced ZEB1 upregulation was attributed to phosphorylation-mediated stability.

AMPK is considered a key regulator of energy metabolism and is activated in the event of energy imbalance, such as lack of energy substrates, energy or oxygen [36,37]. Recent literature reveals that AMPK can exert tumor-promoting or anti-tumor functions in cancer depending on context [38]. On the one hand, activated p-AMPK can inhibit cell growth by participating in p53-dependent cell cycle arrest and down-regulation of mTORC1 activity [38]. On the other hand, AMPK activation can also play a role in promoting tumors in metabolic stress by participating in other metabolic pathways, including the induction of autophagy and fatty acid oxidation $[26,27,37]$. Of note, it is also reported that AMPK can promote the survival of lung cancer cells through inducing histone deacetylase 8 (HDAC8) phosphorylation under glucose starvation [39]. Coincidently, the protein sequence analysis displayed a common phospho-AMPK substrate motif [LXRXX(pS/pT)] in ZEB1 protein. We hypothesized that ZEB1 might be phosphorylated and stabilized by active AMPK during nutrient deprivation. Coincidently, both knockdown and inhibition of AMPK significantly suppressed expression of ZEB1 protein. Furthermore, we revealed that p-AMPK phosphorylated ZEB1 at Thr220 which led to stabilization of ZEB1. Our studies enrich the connotation of the role of AMPK in nutrient stress-induced EMT.

Numerous evidences indicated that IncRNA plays the role of "recruiting" proteins in cells and acts as the "skeleton" of protein complex. Wu et al demonstrated that IncRNA-SENEBLOC enhanced the combination of p53 and MDM2, contributing to ubiquitin-mediated degradation of p53 protein [40]. Besides, IncRNALnczc3h7a serves as a molecular scaffold to bind to both TRIM25 and activated RIG-I, resulting in the stabilization of the RIG-I-TRIM25 complex at the early stage of viral infection [41]. Meanwhile, the results that RNAse reduced the interaction between AMPK reminded the possibility that IncRNA might mediate such an interaction. Coincidently, our study displayed that ZFAS1 was upregulated during nutrient 
deprivation and facilitated the interaction between ZEB1 and AMPK. Meanwhile, depletion of ZFAS1 obviously decreased stabilization of ZEB1, which was induced under nutrient deprivation. Furthermore, AMPK activator-induced phosphorylation and expression of ZEB1 was reduced by knockdown of ZFAS1, while AMPK inhibitor-decreased phosphorylation and expression of ZEB1 was increased by overexpression of ZFAS1. Meanwhile, migration and invasion of PC cells during nutrient starvation was remarkably inhibited after knockdown of ZFAS1. Furthermore, overexpression of ZFAS1 significantly enhanced metastasis of PC tumor, and further suppressed by knockdown of ZEB1. Therefore, our research indicates that ZFAS1 is a critical IncRNA for tumorigenesis of PC by targeting ZEB1. Although numerous evidences have shown that abnormal expression of ZFAS1 contributes to the development and progression in various cancers [42], our research provides new evidence to support that ZFAS1 might be a universal oncogenic IncRNA.

We further explored the mechanism of ZFAS1 upregulation during nutrient deprivation. Since many IncRNAs had been revealed to form feedback with its target [43-45], and also the online database JASPAR predicted a potential binding sites for ZEB1 on the promoter area of ZFAS1 gene, we presumed that ZFAS1 was transcriptionally regulated by ZEB1. Coincidently, the Chip and luciferase results showed that ZEB1 bound to the promoter of ZFAS1 and depletion of ZEB1 abolished the elevated promoter activity induced by nutrient starvation. The clinical results displayed that ZFAS1 was upregulated in PC tissues and correlated with high expression of ZEB1 and VIM, low expression of E-cad, as well as short overall survival in PC patients. Collectively, these results indicate that ZFAS1/ZEB1 feedback plays a pivotal function in EMT and metastasis of PC.

\section{Conclusions}

Our findings implicate that ZFAS1 is a nutrient stress-adapted oncogenic IncRNA which functions as a molecular scaffold facilitating AMPK-mediated phosphorylation and stabilization of ZEB1 in EMT and metastasis of PC cells.

\section{Abbreviations}

PC: pancreatic cancer; EMT: epithelial-mesenchymal transition; ZEB1: zinc finger E-box binding homeobox 1; VIM: Vimentin; E-cad: E-cadherin; PDAC: pancreatic ductal adenocarcinoma; TME: tumor microenvironment; OS: overall survival; IHC: Immunohistochemistry; RT-qPCR: Reverse transcription quantitative polymerase chain reaction; Co-IP: co-immunoprecipitation; C.C: Compound C; ZFAS1: ZNFX1 antisense RNA 1; RIP: RNA immunoprecipitation; COAD: Colon adenocarcinoma; READ: Rectum adenocarcinoma; LIHC: Liver hepatocellular carcinoma; DLBC: Lymphoid Neoplasm Diffuse Large B-cell Lymphoma; THYM: Thymoma; GBM: Glioblastoma multiforme; LGG: Lower Grade Glioma; UCS: Uterine Carcinosarcoma; KIRC: Kidney renal clear cell carcinoma; TGCT: Testicular Germ Cell Tumors; HCC: hepatocellular carcinoma.

\section{Declarations}


Ethics approval and consent to participate

All animal experiments were approved by the Animal Ethics Committee of Tongji Medical College, Huazhong University of Science \& Technology. Human tissues study was approved by the Ethics Committee at the Academic Medical Center of Huazhong University of Science and Technology.

\section{Consent for publication}

Written informed consent was gotten from all patients.

\section{Availability of data and materials}

The data generated or analyses during this study are included in this article and additional files.

\section{Competing interests}

The authors declare that they have no competing interests.

\section{Funding}

The National Science Foundation Committee of China (Grant number: 81372666,81672406 to G.Z.); and Clinical Research Physician Program of Tongji Medical College, Huazhong University of Science and Technology to G.Z. supported this study.

\section{Authors' contributions}

The specific contributions made by all authors are listed below. Gang Zhao: Designed and supervised the research. Zhu Zeng, Shengbo Han, Yan Huang, Yuhang Hu, Fengyu Xu, Yong Zhao, Mengqi Huang, Fan Wang, Jiang Tang and Decai Wang: Performed cellular experiments. Zhu Zeng, Shengbo Han and Yan Huang: Conducted animal experiments. Yang Wang: Provided the clinical data. Gang Zhao and Zhu Zeng: Analyzed the data. Gang Zhao and Zhu Zeng: Wrote the manuscript. All authors read and approved the final manuscript.

\section{Acknowledgements}

We appreciate Jinghui Zhang for providing technical assistance.

\section{References}

1. Siegel RL, Miller KD, Jemal A. Cancer statistics, 2020. CA Cancer J Clin. 2020;70:7-30.

2. Rahib L, Smith BD, Aizenberg R, Rosenzweig AB, Fleshman JM, Matrisian LM. Projecting cancer incidence and deaths to 2030: the unexpected burden of thyroid, liver, and pancreas cancers in the United States. Cancer Res. 2014;74:2913-21. 
3. Loveday BPT, Lipton L, Thomson BN. Pancreatic cancer: An update on diagnosis and management. Aust J Gen Pract. 2019;48(12):826-831.

4. Pereira SP, Oldfield L, Ney A, Hart PA, Keane MG, Pandol SJ, et al. Early detection of pancreatic cancer. Lancet Gastroenterol Hepatol. 2020;5(7):698-710.

5. Conroy T, Desseigne F, Ychou M, Bouche O, Guimbaud R, Becouarn Y, et al. FOLFIRINOX versus gemcitabine for metastatic pancreatic cancer. N Engl J Med. 2011;364:1817-25.

6. Von Hoff DD, Ervin T, Arena FP, Chiorean EG, Infante J, Moore M, et al. Increased survival in pancreatic cancer with nab-paclitaxel plus gemcitabine. N Engl J Med. 2013;369:1691-703.

7. Feig C, Gopinathan A, Neesse A, Chan DS, Cook N, Tuveson DA. The pancreas cancer microenvironment. Clin Cancer Res. 2012;18:4266-76.

8. Hessmann E, Buchholz SM, Demir IE, Singh SK, Gress TM, Ellenrieder V, et al. Microenvironmental Determinants of Pancreatic Cancer. Physiol Rev. 2020;100(4):1707-1751.

9. Ho WJ, Jaffee EM, Zheng L. The tumour microenvironment in pancreatic cancer - clinical challenges and opportunities. Nat Rev Clin Oncol. 2020;17(9):527-540.

10. Otto AM. Metabolic Constants and Plasticity of Cancer Cells in a Limiting Glucose and Glutamine Microenvironment-A Pyruvate Perspective. Front Oncol. 2020;10:596197.

11. Kamphorst JJ, Nofal M, Commisso C, Hackett SR, Lu W, Grabocka E, et al. Human pancreatic cancer tumors are nutrient poor and tumor cells actively scavenge extracellular protein. Cancer Res. 2015;75:544-53.

12. Pastushenko I, Blanpain C. EMT Transition States during Tumor Progression and Metastasis. Trends Cell Biol. 2019;29(3):212-226.

13. Tiwari N, Gheldof A, Tatari M, Christofori G. EMT as the ultimate survival mechanism of cancer cells. Semin Cancer Biol. 2012;22(3):194-207.

14. Jiang J, Tang YL, Liang XH. EMT: a new vision of hypoxia promoting cancer progression. Cancer Biol Ther. 2011;11(8):714-23.

15. Yuen A, Díaz B. The impact of hypoxia in pancreatic cancer invasion and metastasis. Hypoxia (Auckl). 2014;2:91-106.

16. Jo H, Lee J, Jeon J, Kim SY, Chung JI, Ko HY, et al. The critical role of glucose deprivation in epithelial-mesenchymal transition in hepatocellular carcinoma under hypoxia. Sci Rep. 2020;10(1):1538.

17. Recouvreux MV, Moldenhauer MR, Galenkamp KMO, Jung M, James B, Zhang Y, Lowy A, Bagchi A, Commisso C. Glutamine depletion regulates Slug to promote EMT and metastasis in pancreatic cancer. J Exp Med. 2020;217(9):e20200388.

18. Bhan A, Soleimani M, Mandal SS. Long Noncoding RNA and Cancer: A New Paradigm. Cancer Res. 2017;77(15):3965-3981.

19. Deng SJ, Chen HY, Zeng Z, Deng S, Zhu S, Ye Z, et al. Nutrient Stress-Dysregulated Antisense IncRNA GLS-AS Impairs GLS-Mediated Metabolism and Represses Pancreatic Cancer Progression. Cancer 
Res. 2019;79:1398-412.

20. Liu X, Xiao ZD, Han L, Zhang J, Lee SW, Wang W, et al. LncRNA NBR2 engages a metabolic checkpoint by regulating AMPK under energy stress. Nat Cell Biol. 2016;18:431-42.

21. Chen X, Gao Y, Li D, Cao Y, Hao B. LncRNA-TP53TG1 Participated in the Stress Response Under Glucose Deprivation in Glioma. J Cell Biochem. 2017;118(12):4897-4904.

22. Zeng Z, Xu F, Zheng H, Cheng P, Chen Q, Ye Z, et al. LncRNA-MTA2TR functions as a promoter in pancreatic cancer via driving deacetylation-dependent accumulation of HIF-1a. Theranostics. 2019;9:5298-314.

23. Qin Y, Yu J, Zhang M, Qin F, Lan X. ZEB1 promotes tumorigenesis and metastasis in hepatocellular carcinoma by regulating the expression of vimentin. Mol Med Rep. 2019;19:2297-306.

24. Sanchez-Tillo E, Lazaro A, Torrent R, Cuatrecasas M, Vaquero EC, Castells A, et al. ZEB1 represses Ecadherin and induces an EMT by recruiting the SWI/SNF chromatin-remodeling protein BRG1. Oncogene. 2010;29:3490-500.

25. Lee CW, Wong LL, Tse EY, Liu HF, Leong VY, Lee JM, et al. AMPK promotes p53 acetylation via phosphorylation and inactivation of SIRT1 in liver cancer cells. Cancer Res. 2012;72:4394-404.

26. Kim J, Kundu M, Viollet B, Guan KL. AMPK and mTOR regulate autophagy through direct phosphorylation of Ulk1. Nat Cell Biol. 2011;13:132-41.

27. Gwinn DM, Shackelford DB, Egan DF, Mihaylova MM, Mery A, Vasquez DS, et al. AMPK phosphorylation of raptor mediates a metabolic checkpoint. Mol Cell. 2008;30:214-26.

28. Zhou G, Myers R, Li Y, Chen Y, Shen X, Fenyk-Melody J, et al. Role of AMP-activated protein kinase in mechanism of metformin action. J Clin Invest. 2001;108(8):1167-74.

29. Schmitz SU, Grote P, Herrmann BG. Mechanisms of long noncoding RNA function in development and disease. Cell Mol Life Sci. 2016;73(13):2491-509.

30. Livi CM, Klus P, Delli Ponti R, Tartaglia GG. catRAPID signature: identification of ribonucleoproteins and RNA-binding regions. Bioinformatics. 2016;32(5):773-5.

31. Muppirala UK, Honavar VG, Dobbs D. Predicting RNA-protein interactions using only sequence information. BMC Bioinformatics. 2011;12:489.

32. Mittal V. Epithelial Mesenchymal Transition in Tumor Metastasis. Annu Rev Pathol. 2018;13:395-412.

33. Krebs AM, Mitschke J, Lasierra LM, Schmalhofer O, Boerries M, Busch H, et al. The EMT-activator Zeb1 is a key factor for cell plasticity and promotes metastasis in pancreatic cancer. Nat Cell Biol. 2017; 19:518-29.

34. Omenn GS, Lane L, Lundberg EK, Beavis RC, Overall CM, Deutsch EW. Metrics for the Human Proteome Project 2016: Progress on Identifying and Characterizing the Human Proteome, Including Post-Translational Modifications. J Proteome Res. 2016;15(11):3951-3960.

35. Zhang P, Wei Y, Wang L, Debeb BG, Yuan Y, Zhang J, et al. ATM-mediated stabilization of ZEB1 promotes DNA damage response and radioresistance through CHK1. Nat Cell Biol. 2014;16:864-75. 
36. Motoshima H, Goldstein BJ, Igata M, Araki E. AMPK and cell proliferation-AMPK as a therapeutic target for atherosclerosis and cancer. J Physiol. 2006;574:63-71.

37. McFadden JW, Corl BA. Activation of AMP-activated protein kinase (AMPK) inhibits fatty acid synthesis in bovine mammary epithelial cells. Biochem Biophys Res Commun. 2009;390:388-93.

38. Faubert B, Vincent EE, Poffenberger MC, Jones RG. The AMP-activated protein kinase (AMPK) and cancer: many faces of a metabolic regulator. Cancer Lett. 2015;356(2 Pt A):165-70.

39. Li Y, Liang R, Sun M, Li Z, Sheng H, Wang J, et al. AMPK-dependent phosphorylation of HDAC8 triggers PGM1 expression to promote lung cancer cell survival under glucose starvation. Cancer Lett. 2020;478:82-92.

40. Xu CL, Sang B, Liu GZ, Li JM, Zhang XD, Liu LX, et al. SENEBLOC, a long non-coding RNA suppresses senescence via p53-dependent and independent mechanisms. Nucleic Acids Res. 2020;48(6):30893102.

41. Lin H, Jiang M, Liu L, Yang Z, Ma Z, Liu S, et al. The long noncoding RNA Lnczc3h7a promotes a TRIM25-mediated RIG-I antiviral innate immune response. Nat Immunol. 2019;20:812-23.

42. He A, He S, Li X, Zhou L. ZFAS1: A novel vital oncogenic IncRNA in multiple human cancers. Cell Prolif. 2019;52:e12513.

43. Xu MD, Wang Y, Weng W, Wei P, Qi P, Zhang Q, et al. A Positive Feedback Loop of IncRNA-PVT1 and FOXM1 Facilitates Gastric Cancer Growth and Invasion. Clin Cancer Res. 2017;23(8):2071-2080.

44. Wang X, Li L, Zhao K, Lin Q, Li H, Xue X, et al. A novel LncRNA HITT forms a regulatory loop with HIF$1 \mathrm{a}$ to modulate angiogenesis and tumor growth. Cell Death Differ. 2020;27(4):1431-1446.

45. Lan T, Yuan K, Yan X, Xu L, Liao H, Hao X, et al. LncRNA SNHG10 Facilitates Hepatocarcinogenesis and Metastasis by Modulating Its Homolog SCARNA13 via a Positive Feedback Loop. Cancer Res. 2019;79(13):3220-3234.

\section{Figures}


Fig. 1
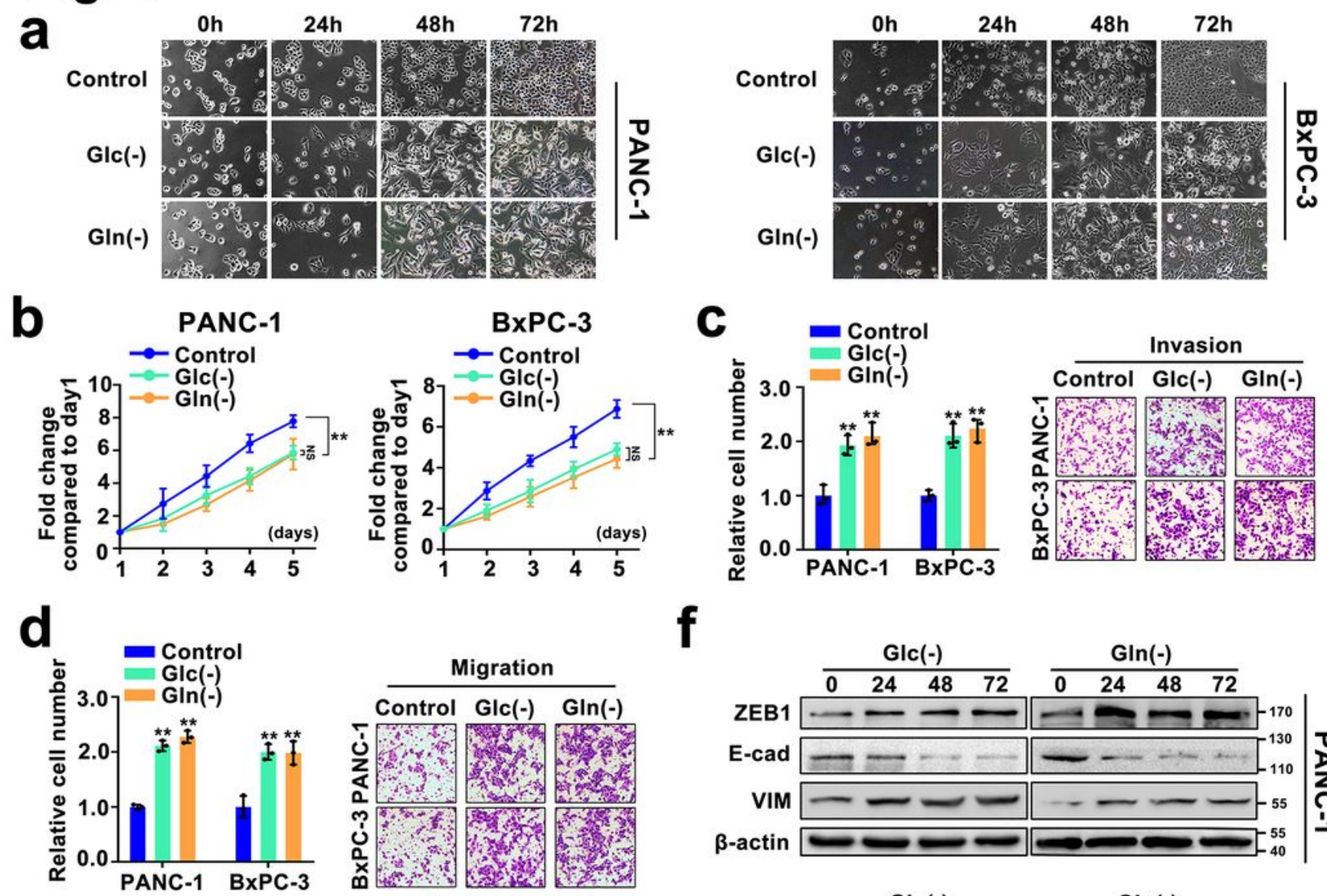

f
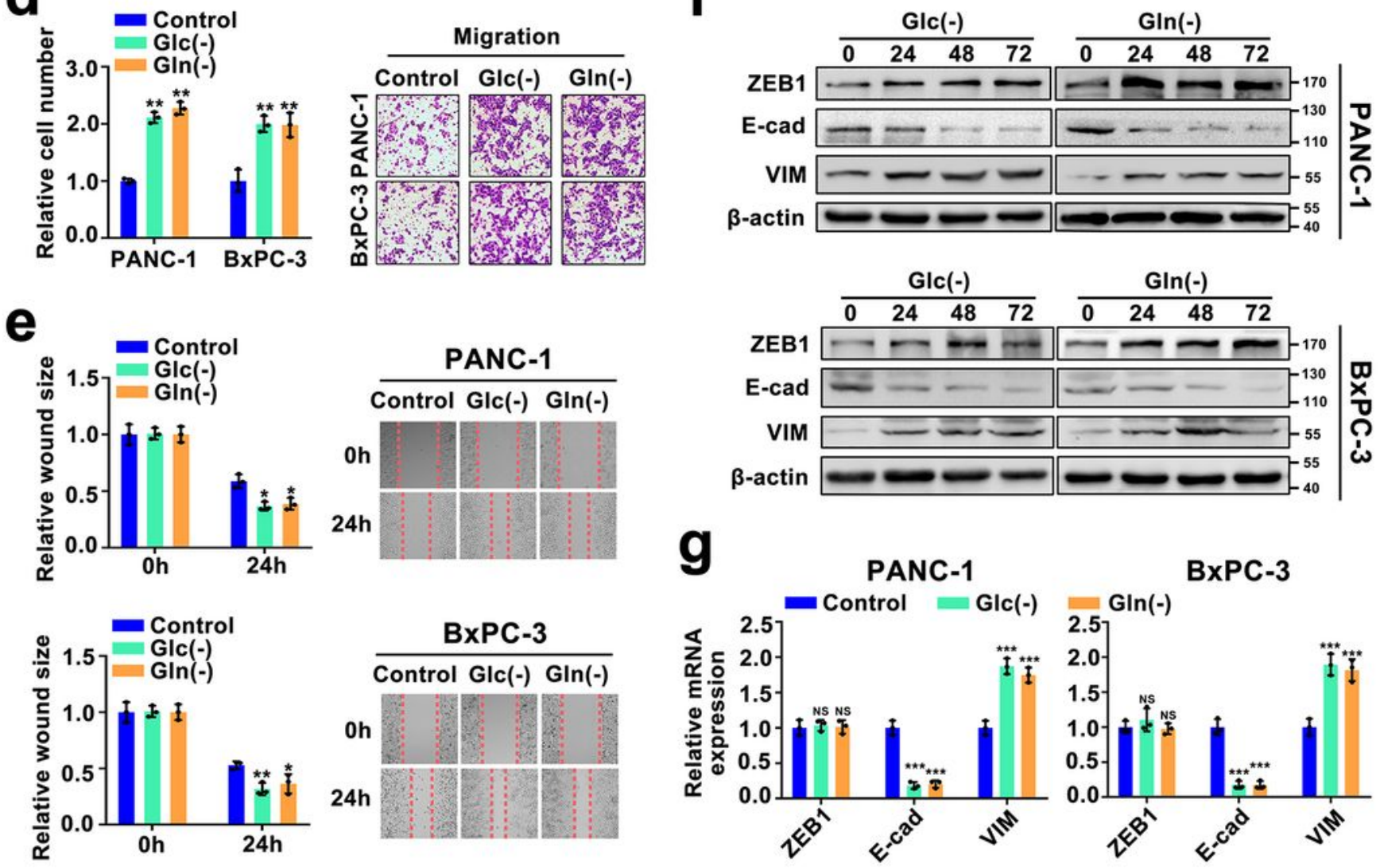

PANC-1

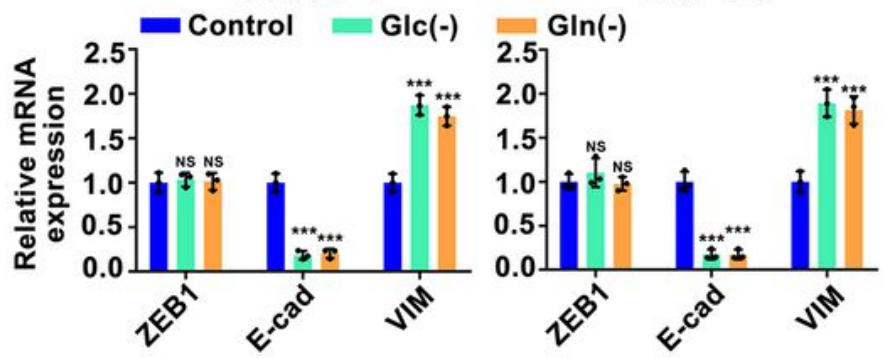

Figure 1

EMT is an adaption of PC cells in response to glucose and glutamine deprivation. a Cell morphology of PANC-1/BxPC-3 cells cultured with saturating nutrient medium, glucose-free (Glc-) medium and glutamine-free (GIn-) medium for 0 h, 24 h, 48 h and 72 h. b Viability of PANC-1/BxPC-3 cells cultured with complete medium, glucose-free medium and glutamine-free medium. c Invasion ability of PANC-1/BxPC-3 cells, cultured with complete medium, glucose-free medium and glutamine-free medium for $48 \mathrm{~h}$, was 
assessed by transwell assay. $d$, e Migration ability of PANC-1/BXPC-3 cells, cultured with complete medium, glucose-free medium and glutamine-free medium for $48 \mathrm{~h}$, was assessed by transwell and wound healing assay. $\mathrm{f}$ After being exposed to glucose-free medium and glutamine-free medium for $0 \mathrm{~h}$, $24 \mathrm{~h}, 48 \mathrm{~h}$ and $72 \mathrm{~h}$, the protein levels of ZEB1, E-cad and VIM were detected by Western Blot in PANC1/BxPC-3 cells. Data shown represent three independent experiments. $g$ After being exposed to complete medium, glucose-free medium and glutamine-free medium for $48 \mathrm{~h}$, the mRNA levels of ZEB1, E-cad and

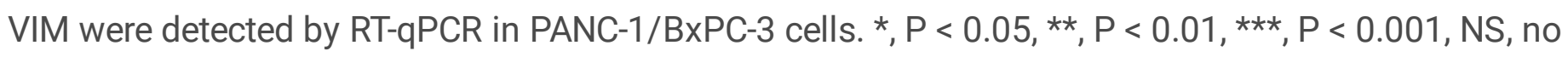
significant. 
a

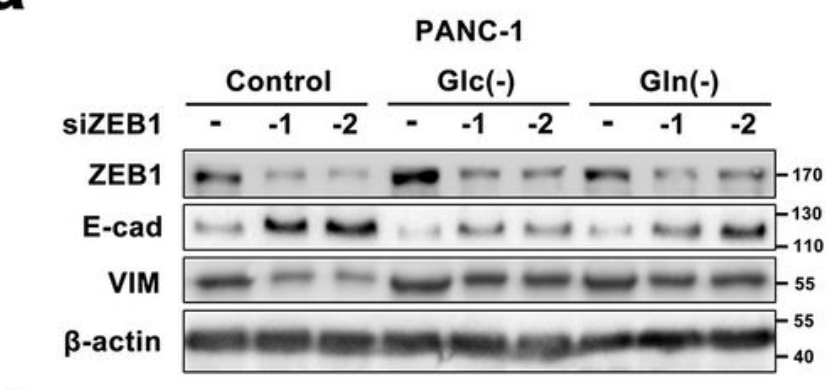

b
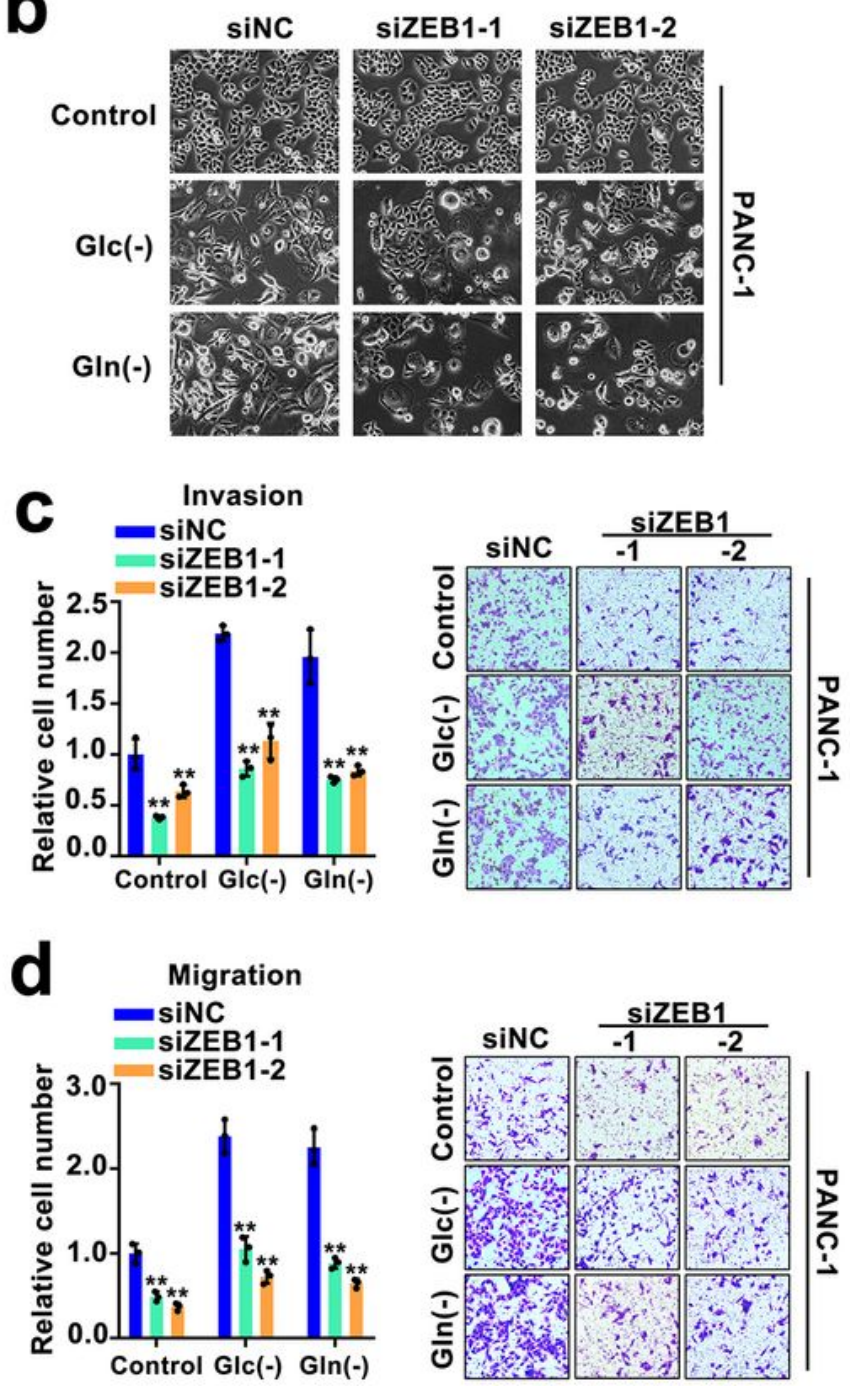
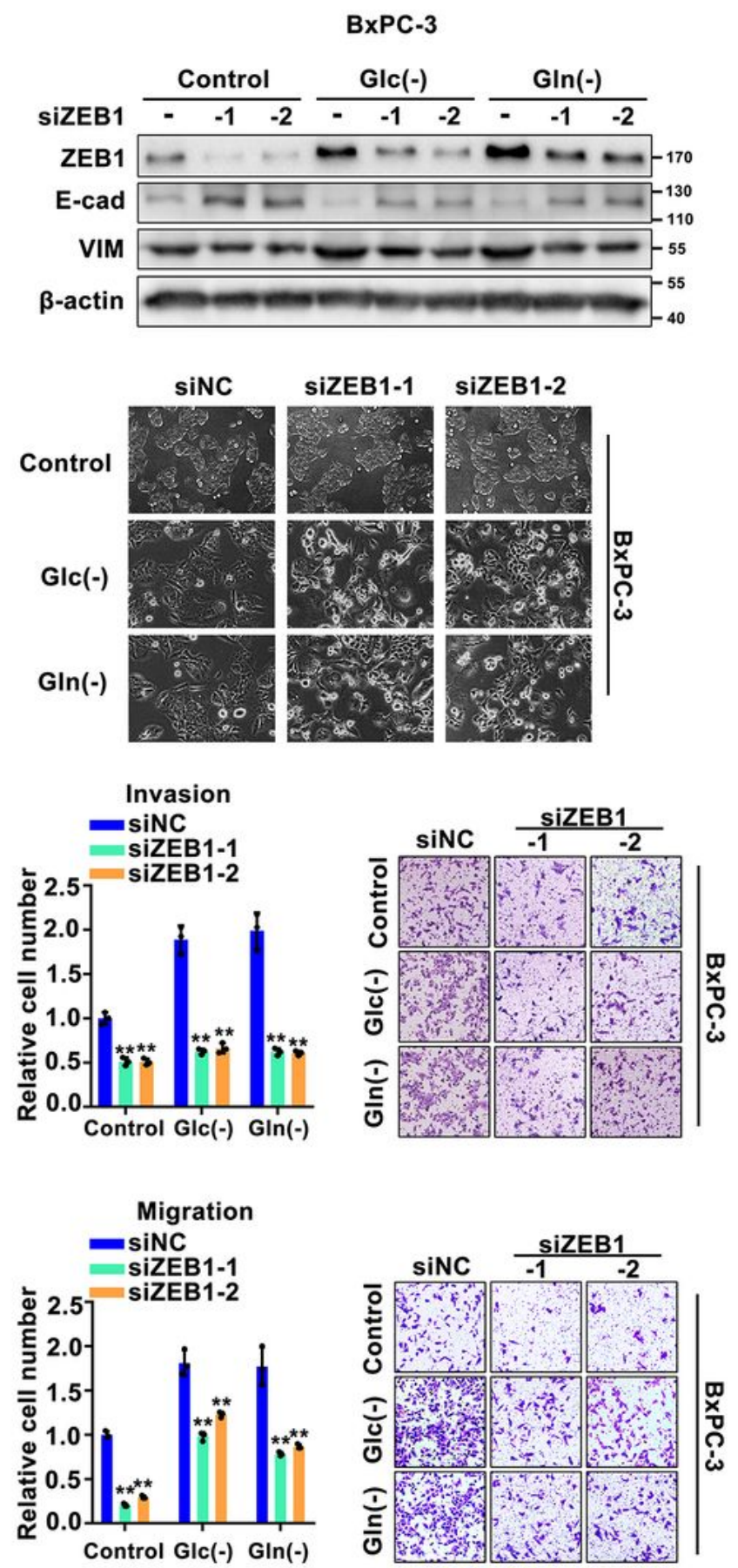

\section{Figure 2}

Upregulated ZEB1 protein is a critical regulator for nutrient starve-induced EMT of PC cells. a-d PANC1/BXPC-3 cells were transfected with siRNA targeting ZEB1 and cultured under complete medium, glucose-free medium and glutamine-free medium for $48 \mathrm{~h}$. a The protein levels of ZEB1, E-cad and VIM were detected by Western Blot. b Cell morphology of ZEB1 knockdown PANC-1/BxPC-3 cells under glucose and glutamine starvation. c Invasion ability of ZEB1 knockdown PANC-1/BxPC-3 cells under 
glucose and glutamine starvation was assessed by transwell assay. $d$ Migration ability of ZEB1 knockdown PANC-1/BxPC-3 cells under glucose and glutamine starvation was assessed by transwell. Data shown represent three independent experiments. *, $\mathrm{P}<0.05,{ }^{\star}$, $\mathrm{P}<0.01$.

\section{Fig. 3}
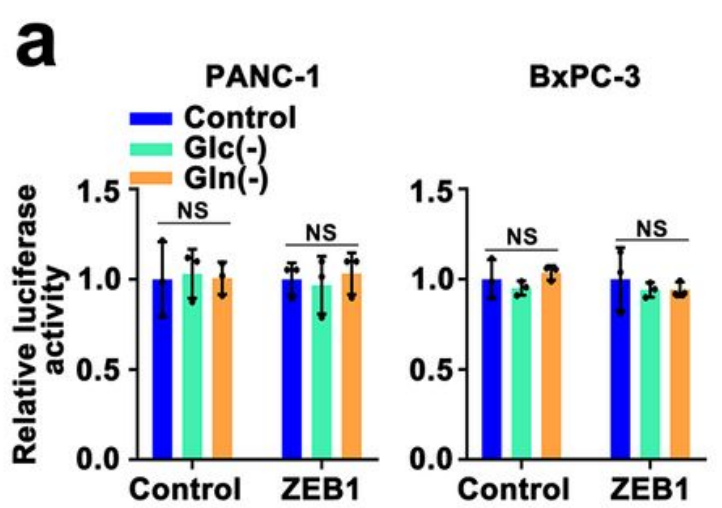

b
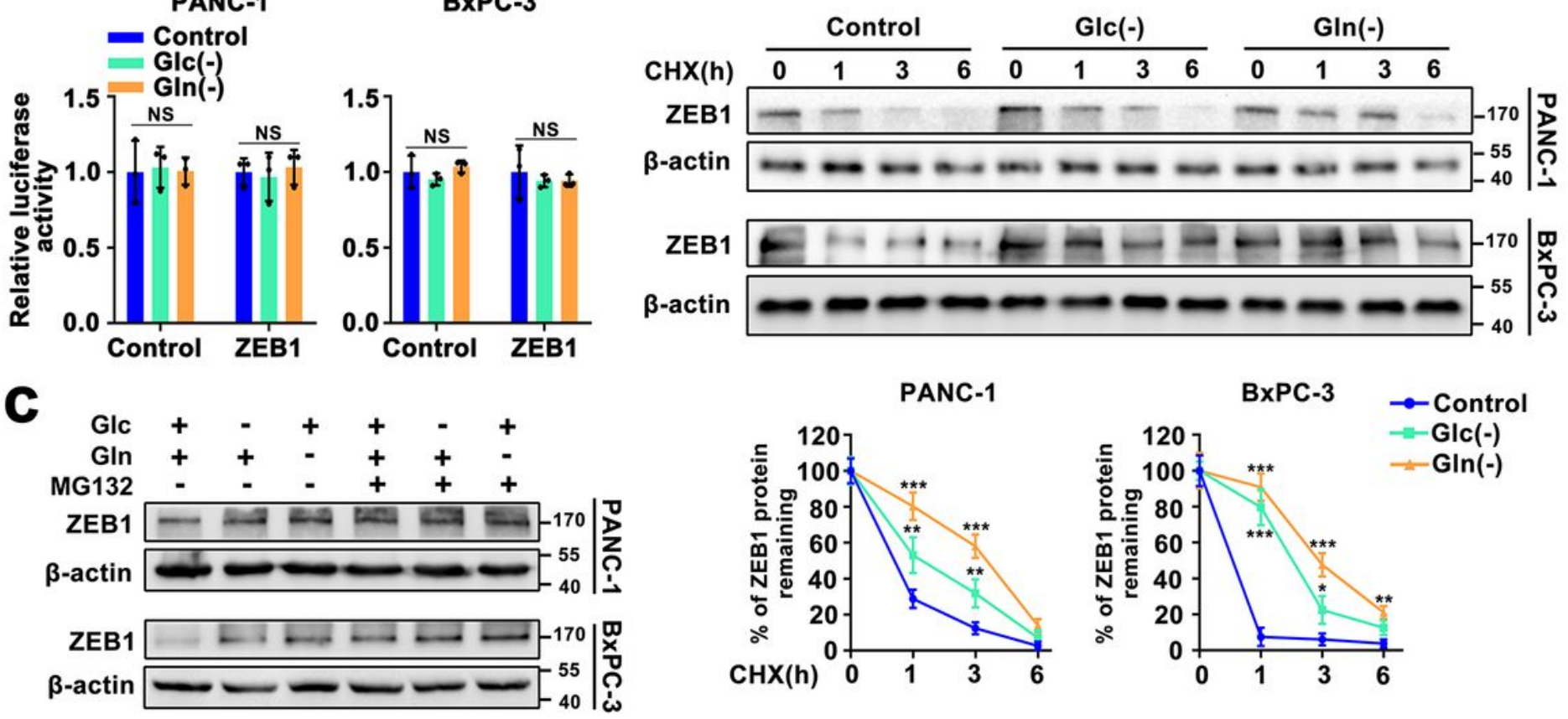

PANC-1
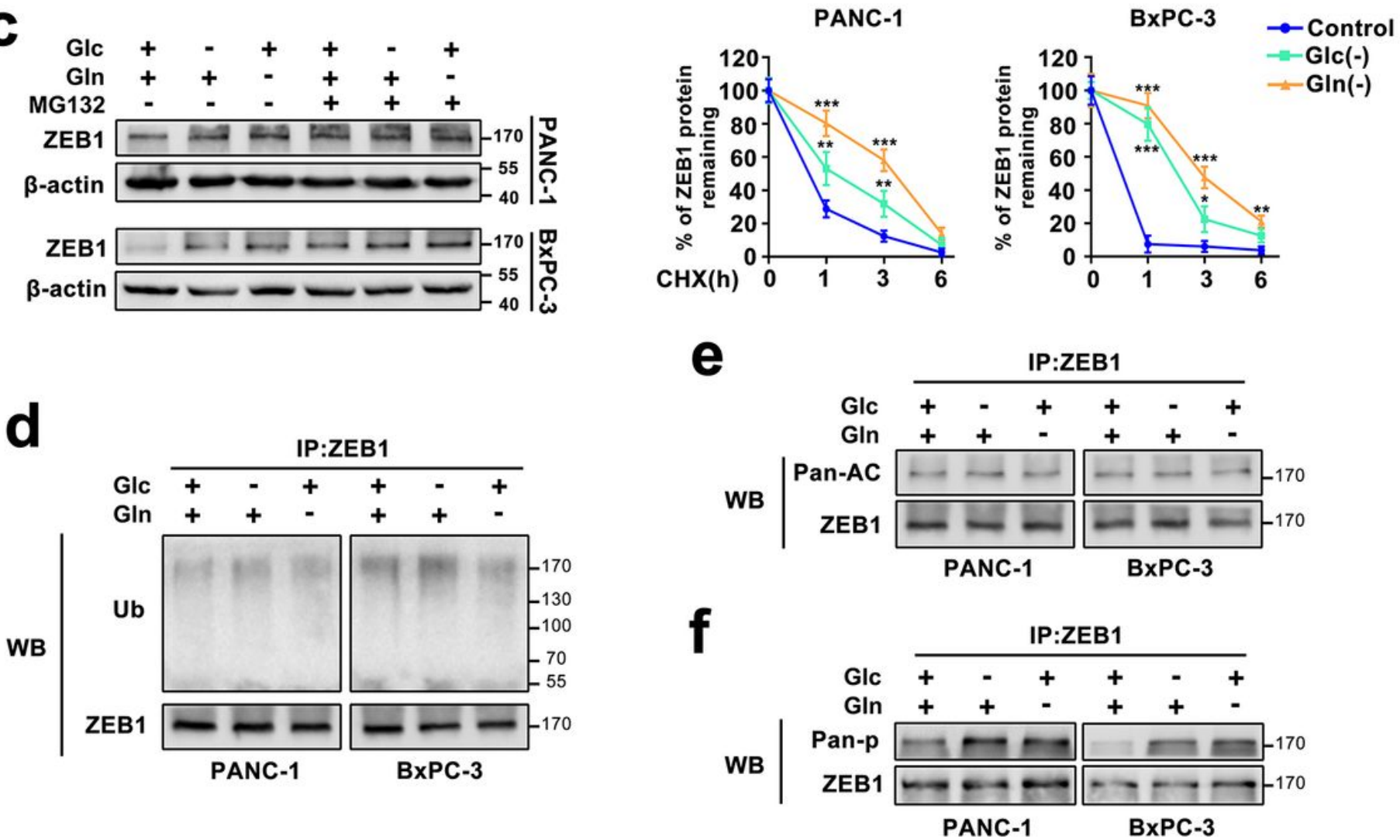

Figure 3

ZEB1 protein was regulated at post-translational level in response to nutrient depletion via phosphorylation modification. a PANC-1/BxPC-3 cells, cultured with complete medium, glucose-free medium and glutamine-free medium, were transfected with pGL3 vector or ZEB1 promoter with firefly 
luciferase plasmid and pRL-TK plasmid encoding Renilla luciferase for $48 \mathrm{~h}$. The luciferase activity of cells was assessed by luciferase reporter assay. b After being cultured with complete medium, glucosefree medium and glutamine-free medium for $48 \mathrm{~h}, \mathrm{PANC}-1 / \mathrm{BxPC}-3$ cells were treated with cycloheximide $(\mathrm{CHX}, 50 \mu \mathrm{g} / \mathrm{ml})$ for periods of time as indicated. Then extracting the total proteins and analyzing the protein level of ZEB1 by Western Blot. The line chart quantifies the relative density of protein above. $C$ Western Blot assay analyzed the protein level of ZEB1 in PANC-1/BXPC-3 cells cultured with complete medium, glucose-free medium and glutamine-free medium for $48 \mathrm{~h}$ with or without the absence of MG132 (20 $\mu \mathrm{M})$ for 3 h. d-f Ubiquitination (d), acetylation (e) and phosphorylated (f) of ZEB1, detected in PANC-1/BXPC-3 cells cultured with complete medium, glucose-free medium and glutamine-free medium for $48 \mathrm{~h}$, were analyzed in the cell lysates following anti-ZEB1 immunoprecipitation. ${ }^{*}, \mathrm{P}<0.05,{ }^{\star}, \mathrm{P}<$ $0.01, \star \star \star, P<0.001, N S$, no significant. 
Fig. 4

a

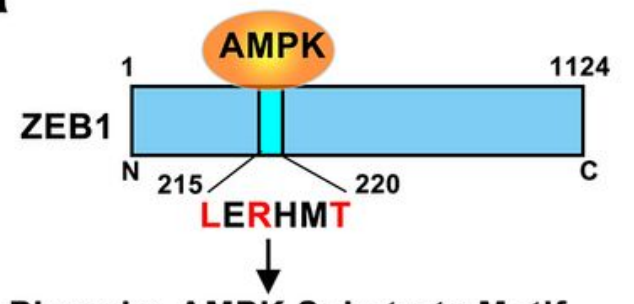

Phospho-AMPK Substrate Motif [LXRXX(pS/pT)]

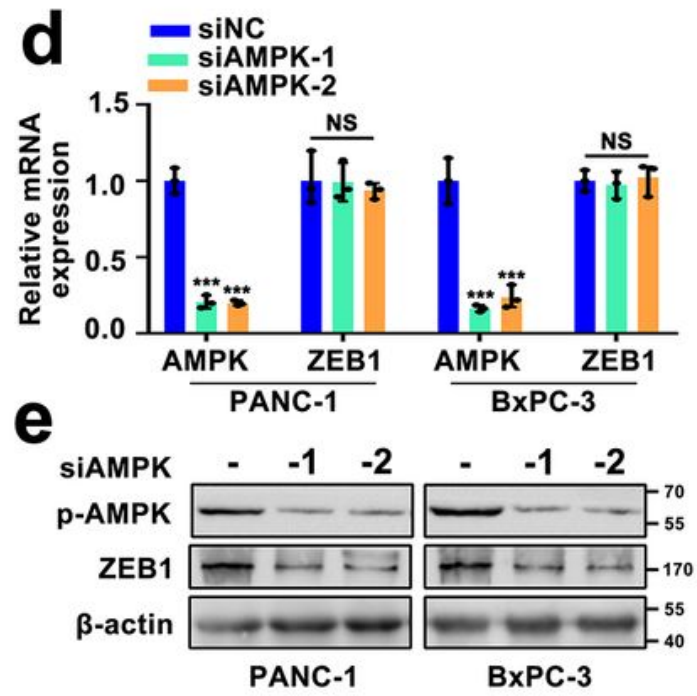

g
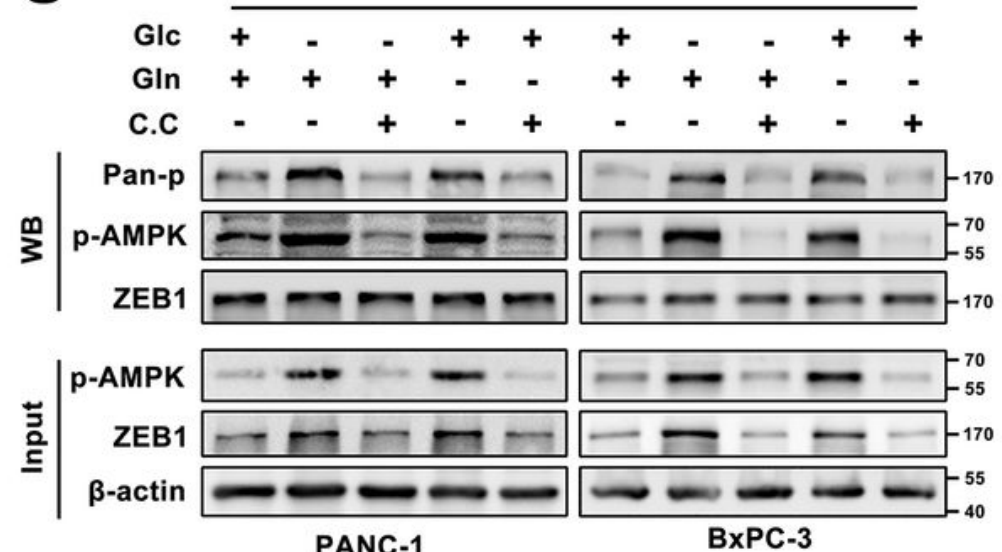

PANC-1
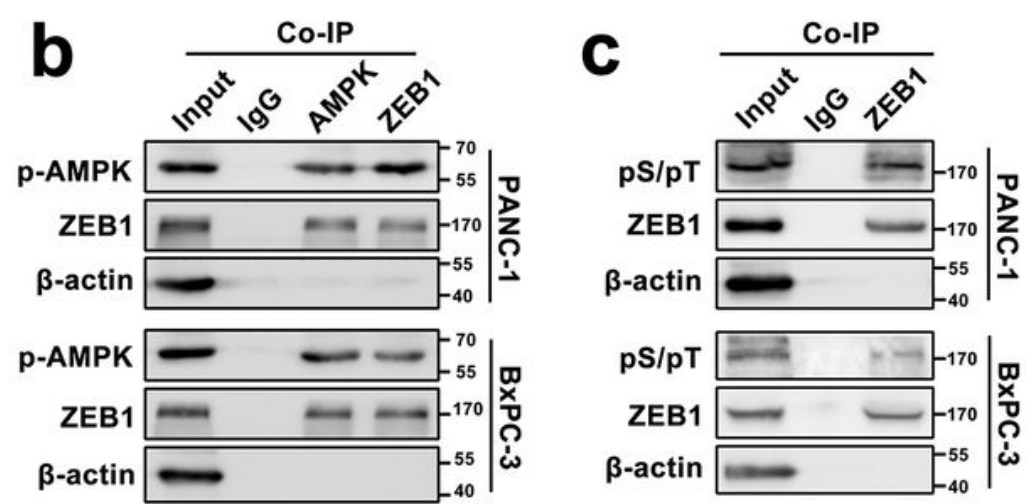

IP:ZEB1

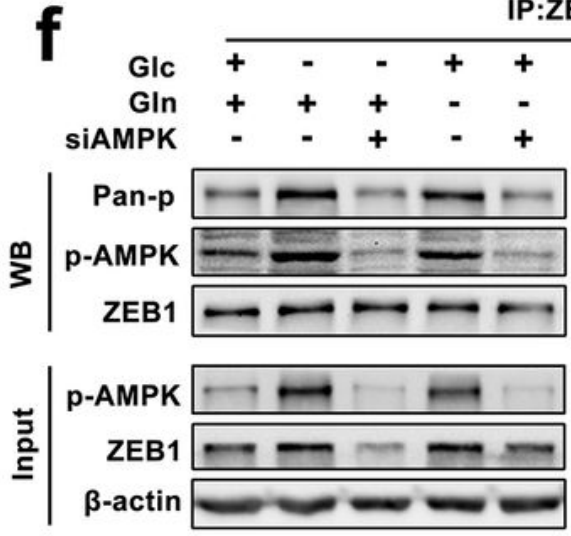

PANC-1

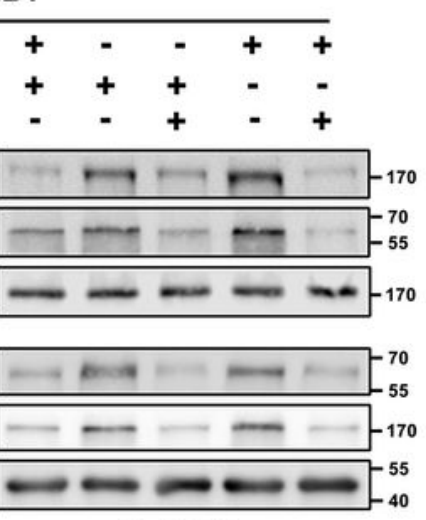

BxPC-3
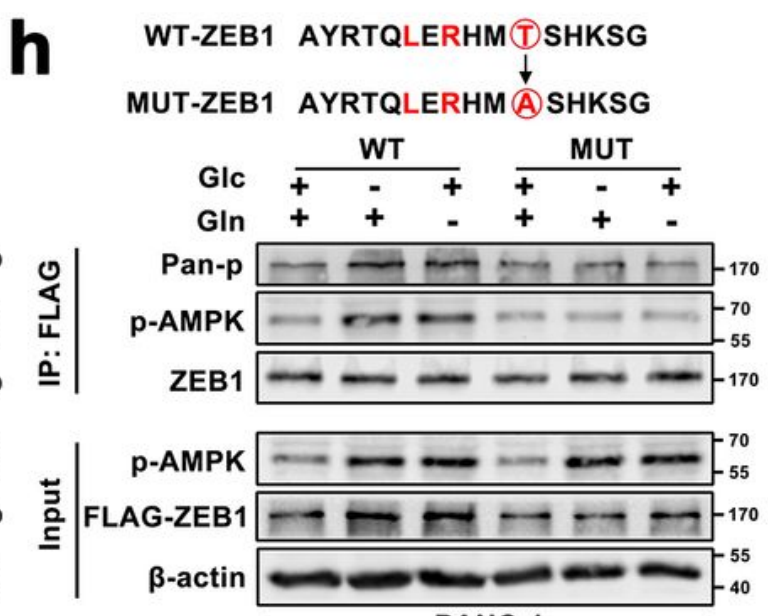

PANC-1

Figure 4

AMPK-dependent phosphorylation at Thr220 mediates the stabilization of ZEB1 under nutrient starvation. a Schematic model presenting the potential phosphorylation sites of AMPK on ZEB1 protein sequence. $b$ AMPK, ZEB1 and $\beta$-actin protein levels were detected in PANC-1/BxPC-3 cell lysates following anti-ZEB1 or anti-AMPK immunoprecipitation. c Proteins, detected with phospho-AMPK substrate motif antibody (pS/pT), ZEB1 and $\beta$-actin antibody in PANC-1/BxPC-3 cells, were analyzed in the cell lysates following 
anti-ZEB1 immunoprecipitation. $d$ The mRNA level of AMPK and ZEB1 were detected by RT-qPCR in PANC-1/BxPC-3 cells transfected with siAMPK-1, siAMPK-2 or correlated control. e Western Blot analysis of AMPK and ZEB1 protein level in PANC-1/BXPC-3 cells transfected with siAMPK-1, siAMPK-2 or correlated control. f, g PANC-1/BxPC-3 cells, cultured with complete medium, glucose-free medium and glutamine-free medium, were transfected with siNC/siAMPK or treated with or without C.C $(5 \mu \mathrm{M})$ for 48 h. Phosphorylated ZEB1, phosphorylated AMPK and ZEB1 protein levels were analyzed in the cell lysates following anti-ZEB1 immunoprecipitation. h PANC-1 cells, cultured with complete medium, glucose-free medium and glutamine-free medium, were transfected with the wild type and mutant type (threonine to alanine) of ZEB1 plasmids for $48 \mathrm{~h}$. Phosphorylated ZEB1, phosphorylated AMPK and ZEB1 protein levels were analyzed in the cell lysates following anti-FLAG immunoprecipitation. Data shown represent three independent experiments. ${ }^{\star \star \star}, \mathrm{P}<0.001$, NS, no significant. 
Fig. 5
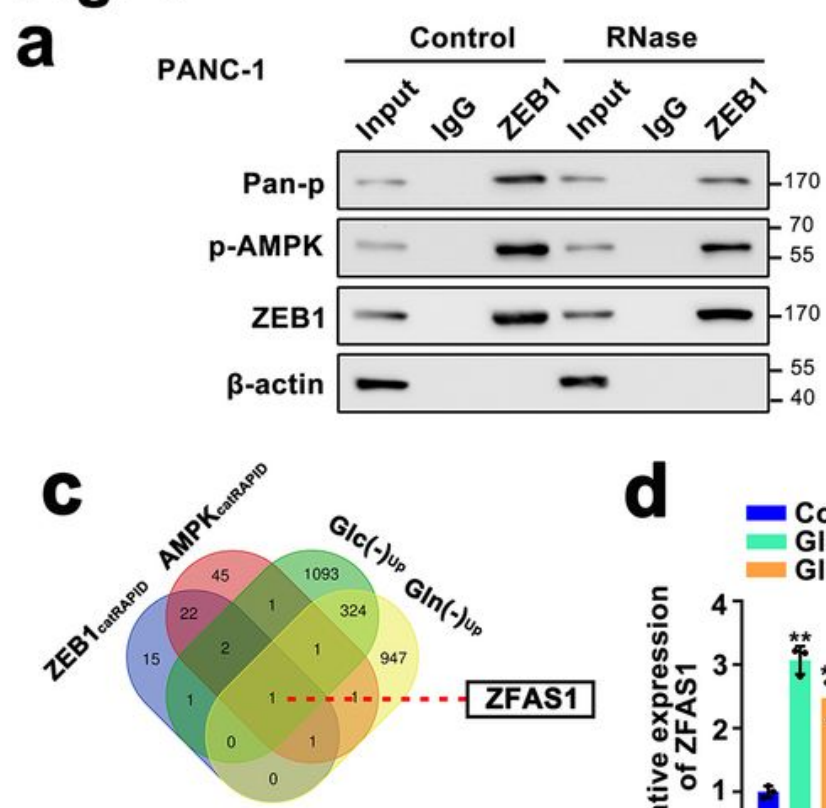

RNA-Protein Interaction Prediction(RPISeq)

\begin{tabular}{|ccc|}
\hline Classifier & RF & SVM \\
\hline AMPK-ZFAS1 & 0.80 & 0.86 \\
\hline ZEB1-ZFAS1 & 0.85 & 0.39 \\
\hline
\end{tabular}
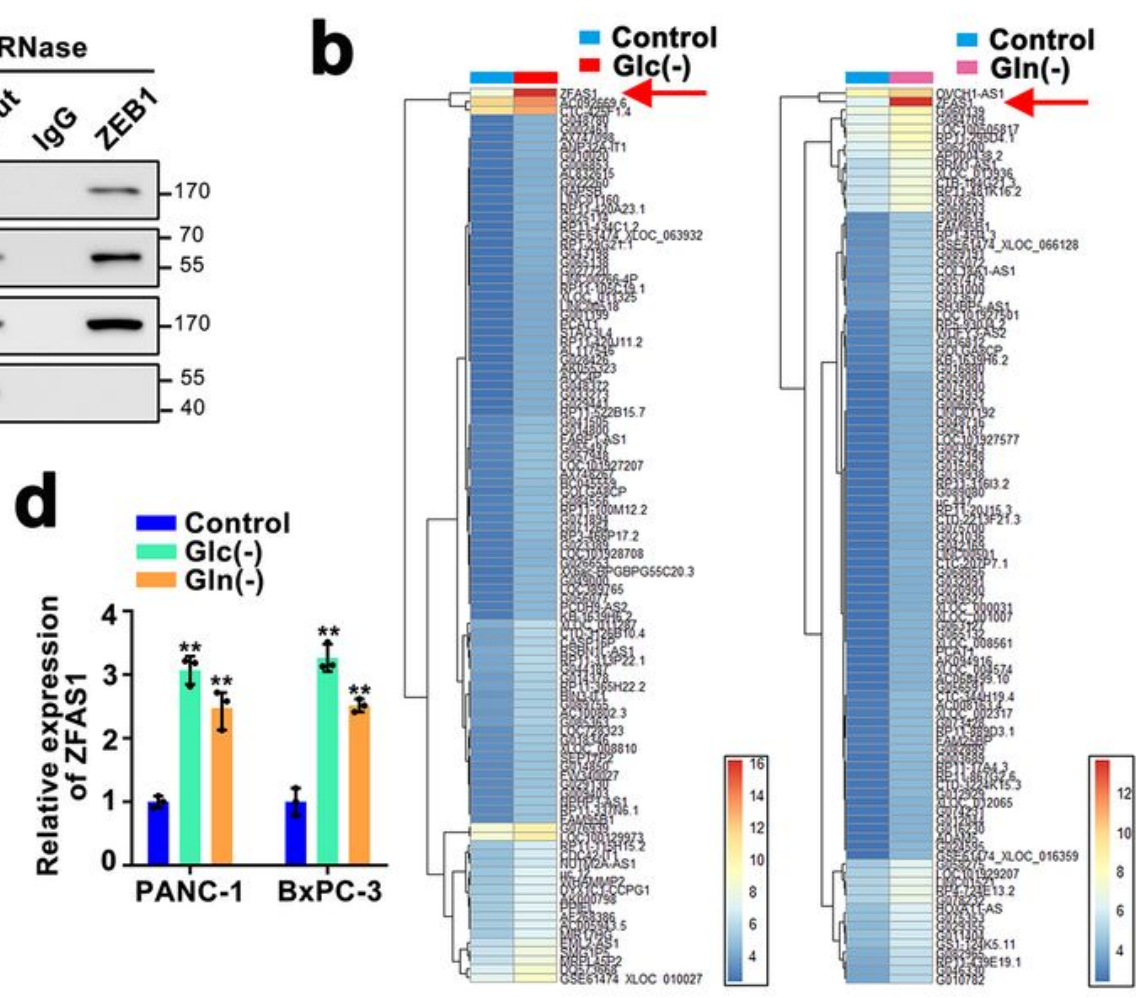

6

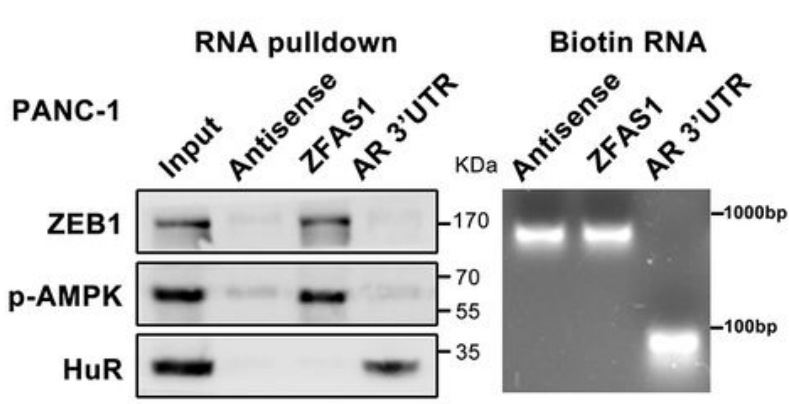

3
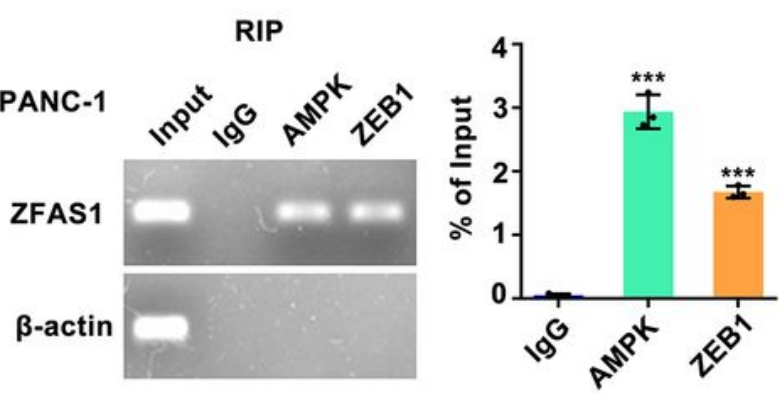

g
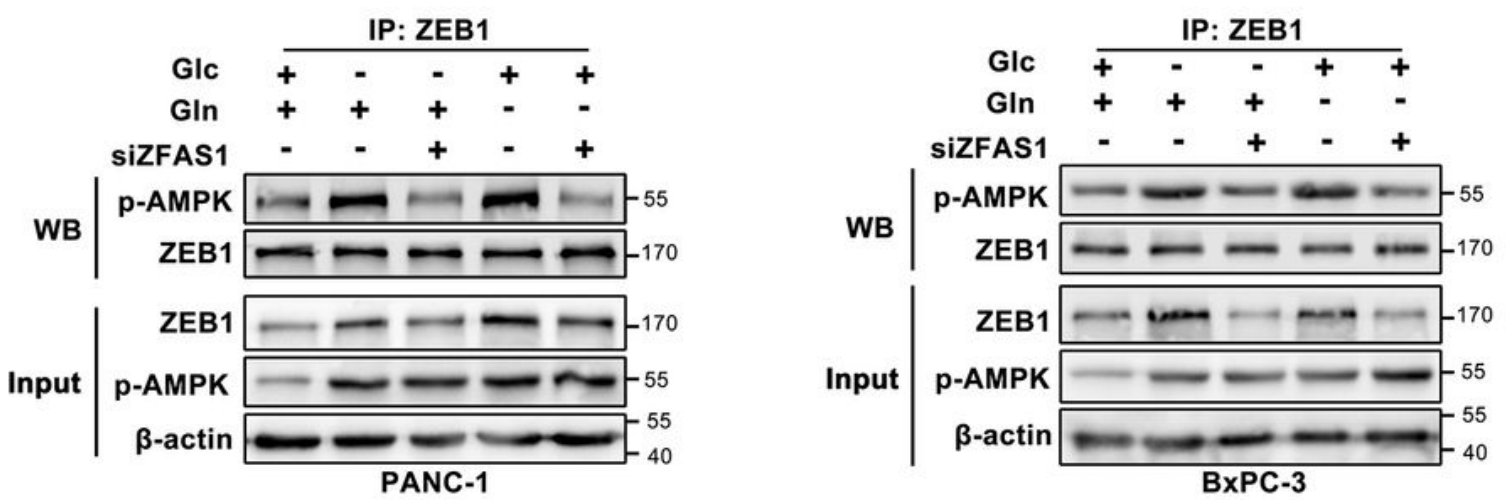

\section{Figure 5}

ZFAS1 facilitates interaction between AMPK and ZEB1. a Protein level of phosphorylated ZEB1, phosphorylated AMPK, ZEB1 and $\beta$-actin were analyzed by Western Blot in the PANC-1 cell lysates treated with or without RNase following anti-ZEB1 immunoprecipitation. b Microarray analysis for IncRNAs was conducted with PANC-1 cells cultured with complete medium, glucose-free medium and glutamine-free medium. The heatmap displayed 100 most upregulated IncRNAs under glucose and glutamine starvation, 
respectively. c (Up) The Venn diagram of the overlap indicated that ZFAS1 was upregulated in both glucose and glutamine free conditions, and may bind to AMPK and ZEB1 predicted through catRAPID database. (Down) RPISeq database predicted the probabilities of the binding between ZFAS1 and AMPK/ZEB1 using Random Forest (RF) and Support Vector Machine (SVM) classifier. $d$ The expression of ZFAS1 was detected by RT-qPCR in PANC-1/BxPC-3 cells cultured with complete medium, glucose-free medium and glutamine-free medium. e RNA pulldown assay was performed to verify the binding between ZFAS1 and ZEB1/AMPK with a biotinylated ZFAS1 probe in PANC-1 cells. Antisense of ZFAS1 and AR 3'UTR served as negative and positive control, respectively. f RIP assay was conducted to verify the binding between ZEB1/AMPK and ZFAS1 with anti-ZEB1 and anti-AMPK antibody in PANC-1 cells. RTqPCR and $2 \%$ agarose gel electrophoresis were performed to detect the ZFAS1 and $\beta$-actin. g PANC1/BxPC-3 cells, cultured with complete medium, glucose-free medium and glutamine-free medium, were transfected with siNC/siZFAS1 for $48 \mathrm{~h}$. Phosphorylated AMPK and ZEB1 protein levels were analyzed in the cell lysates following anti-ZEB1 immunoprecipitation. Data shown represent three independent experiments. ${ }^{* *} p<0.01,{ }^{* \star *} p<0.001$. 
Fig. 6

a

a

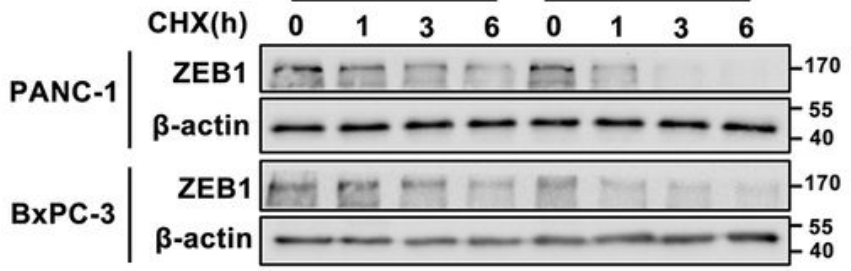

b

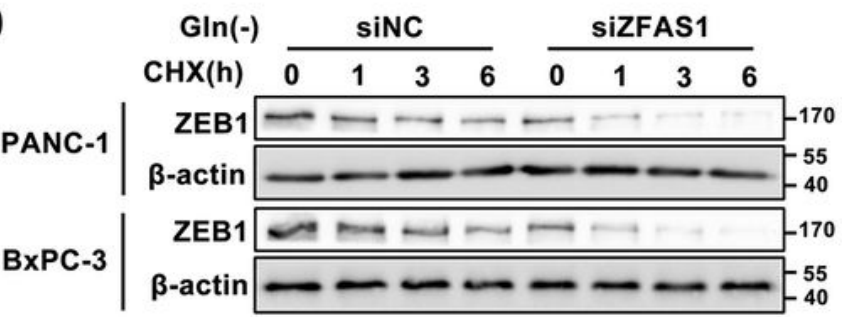

C

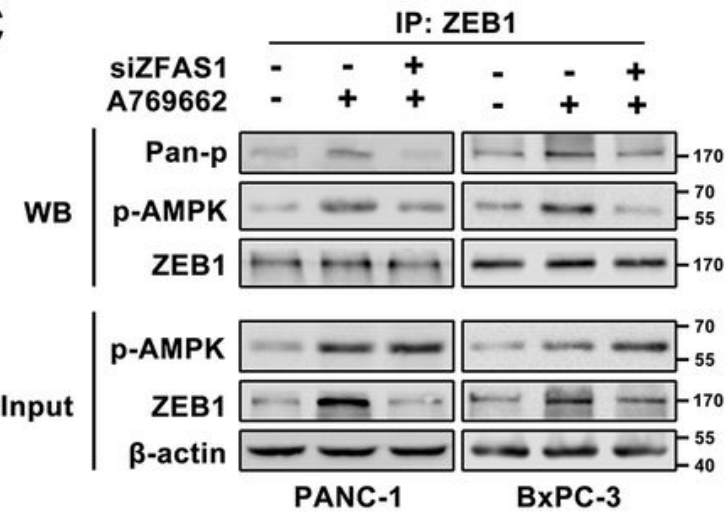

$\mathbf{e}$

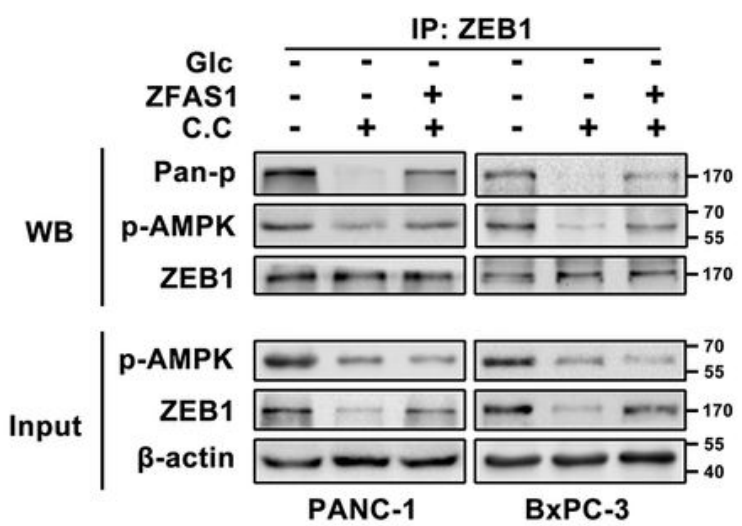

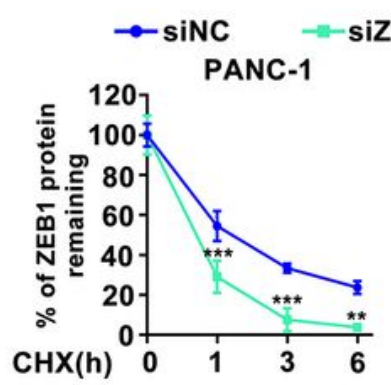
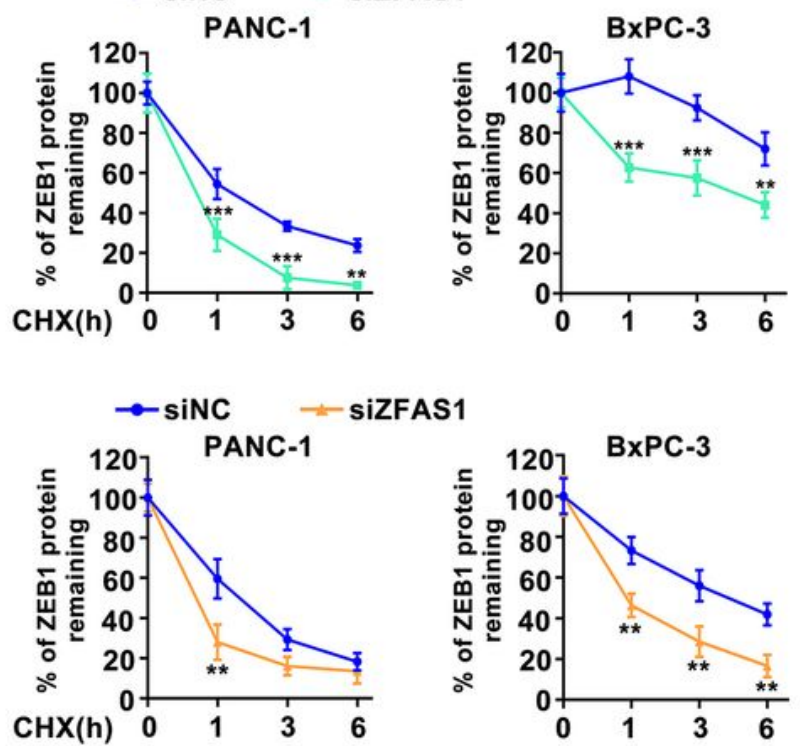

IP: ZEB1

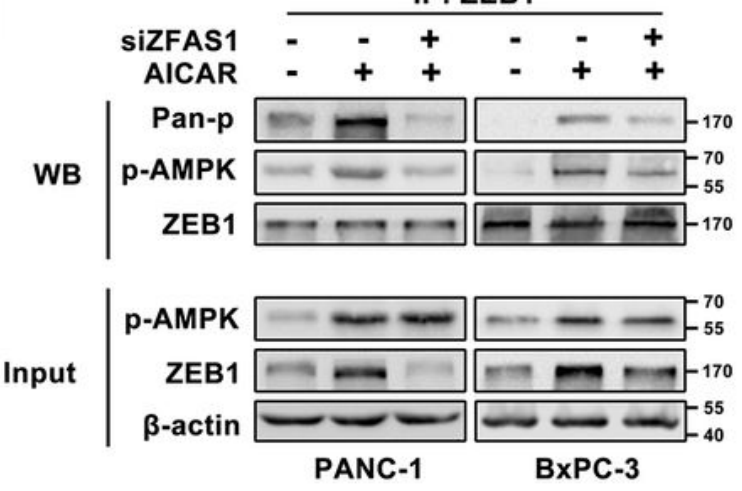

$\mathbf{f}$

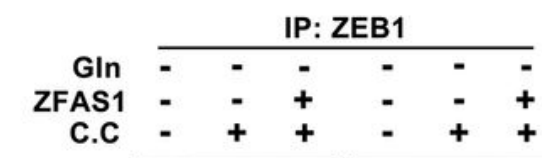

WB

Pan-p $\longrightarrow$ - in -170 p-AMPK - - - - - - - -

ZEB1 $\Longrightarrow \mathrm{FH}$

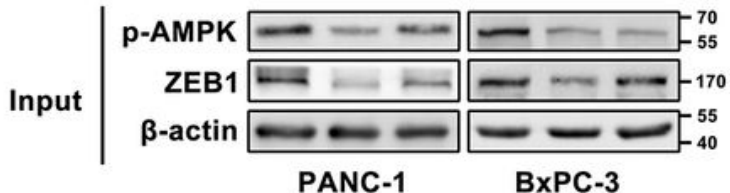

\section{Figure 6}

ZFAS1 enhances ZEB1 protein stability through promoting the AMPK-mediated phosphorylation. $a, b$ After being cultured with glucose-free medium and glutamine-free medium for $48 \mathrm{~h}$, the PANC-1/BxPC-3 cells with ZFAS1 knockdown were treated with cycloheximide ( $\mathrm{CHX}, 50 \mu \mathrm{g} / \mathrm{ml})$ for periods of time as indicated. Then extracting the total proteins and analyzing the protein level of ZEB1 by Western Blot. The line chart quantifies the relative density of protein above. c PANC-1/BxPC-3 cells were transfected with 
siNC/siZFAS1 for $48 \mathrm{~h}$ and treated with or without A769662 (100 mM) for $4 \mathrm{~h}$. Phosphorylated ZEB1, phosphorylated AMPK and ZEB1 protein levels were analyzed in the cell lysates following anti-ZEB1 immunoprecipitation. d PANC-1/BxPC-3 cells were transfected with siNC/siZFAS1 for $48 \mathrm{~h}$ and treated with or without AICAR $(0.5 \mathrm{mM})$ for $5 \mathrm{~h}$. Phosphorylated ZEB1, phosphorylated AMPK and ZEB1 protein levels were analyzed in the cell lysates following anti-ZEB1 immunoprecipitation. e, f PANC-1/BxPC-3 cells, cultured with glucose-free medium (e) or glutamine-free medium (f) for $48 \mathrm{~h}$, were transfected with ZFAS1 overexpression plasmid or treated with or without C.C $(5 \mu \mathrm{M})$ for $48 \mathrm{~h}$. Phosphorylated ZEB1, phosphorylated AMPK and ZEB1 protein levels were analyzed in the cell lysates following anti-ZEB1 immunoprecipitation. Data shown represent three independent experiments. ${ }^{\star \star} p<0.01, \star \star \star p<0.001$.

\section{Fig. 7}
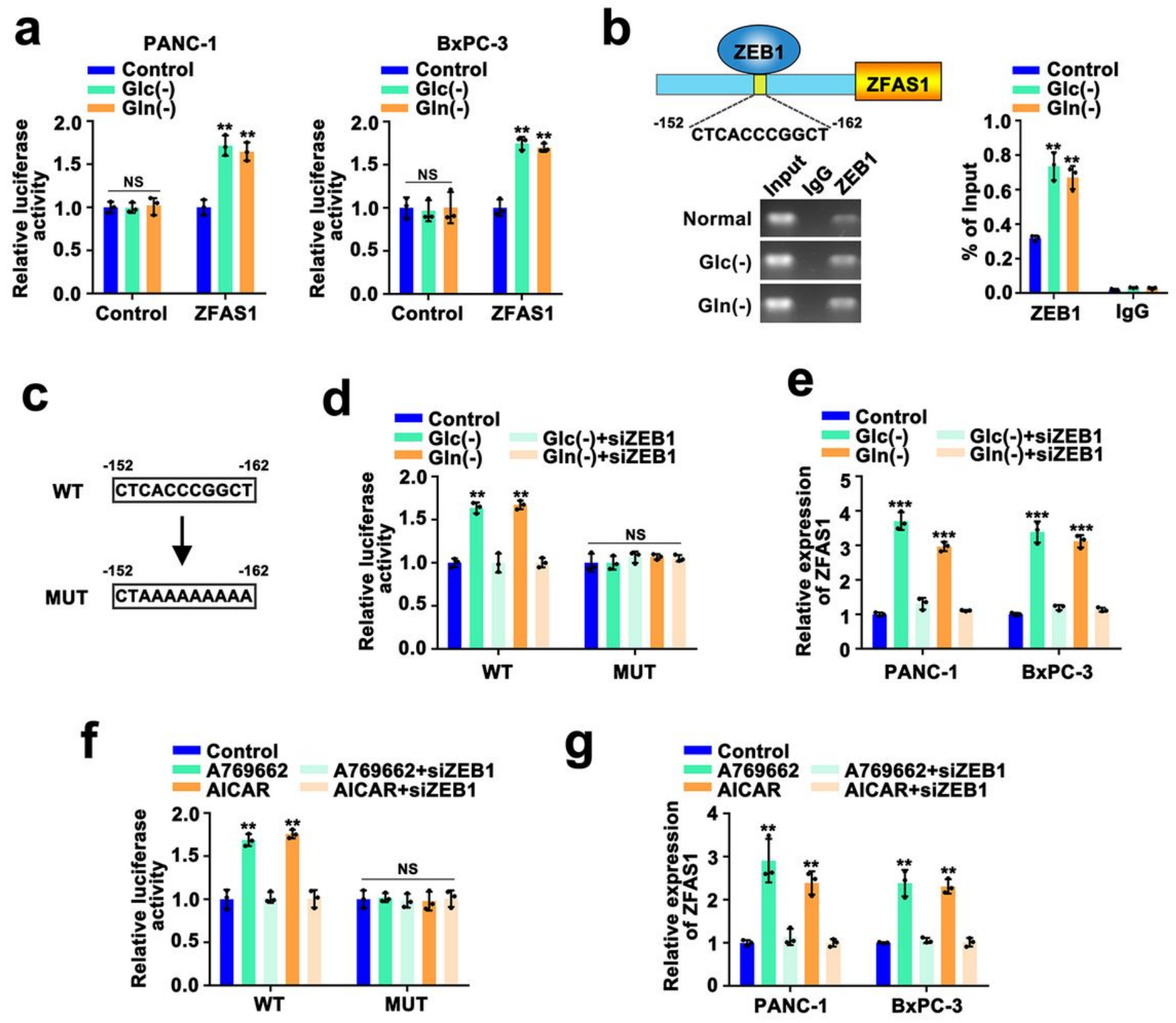


\section{Figure 7}

ZFAS1 is transcriptionally regulated by ZEB1 under glucose and glutamine deprivation. a PANC-1/BxPC-3 cells, cultured with complete medium, glucose-free medium and glutamine-free medium, transfected with pGL3 vector or ZFAS1 promoter with firefly luciferase plasmid and pRL-TK plasmid encoding Renilla luciferase for $48 \mathrm{~h}$. The luciferase activity of cells was assessed by luciferase reporter assay. b Schematic model presenting the potential binding sites of ZEB1 on ZFAS1 promoter sequence. PANC-1 cells were cultured with complete medium, glucose-free medium and glutamine-free medium for $48 \mathrm{~h}$. Then the cells were lysed for the ChIP assay. c Schematic model presenting the wild-type and mutant-type of ZFAS1 promoter sequence. d PANC-1 cells, cultured with complete medium, glucose-free medium and glutaminefree medium, were co-transfected with pGL3 ZFAS1 promoter (wild-type/mutant-type) with firefly luciferase plasmid, pRL-TK plasmid encoding Renilla luciferase and siNC/siZEB1 for $48 \mathrm{~h}$. The luciferase activity of cells was assessed by luciferase reporter assay. e The expression of ZFAS1 was detected by RT-qPCR in PANC-1/BxPC-3 cells transfected with siNC/siZEB1 and cultured with complete medium, glucose-free medium and glutamine-free medium. f PANC-1 cells were co-transfected with pGL3 ZFAS1 promoter (wild-type/mutant-type) with firefly luciferase plasmid, pRL-TK plasmid encoding Renilla luciferase and siNC/siZEB1 for $48 \mathrm{~h}$ and treated with or without A769662/AICAR. The luciferase activity of cells was assessed by luciferase reporter assay. $g$ The expression of ZFAS1 was detected by RT-qPCR in PANC-1/BxPC-3 cells transfected with siNC/siZEB1 for $48 \mathrm{~h}$ and treated with or without A769662/AICAR. Data shown represent three independent experiments. ${ }^{\star *} p<0.01,{ }^{\star \star \star} p<0.001$. NS, no significant. 
Fig. 8
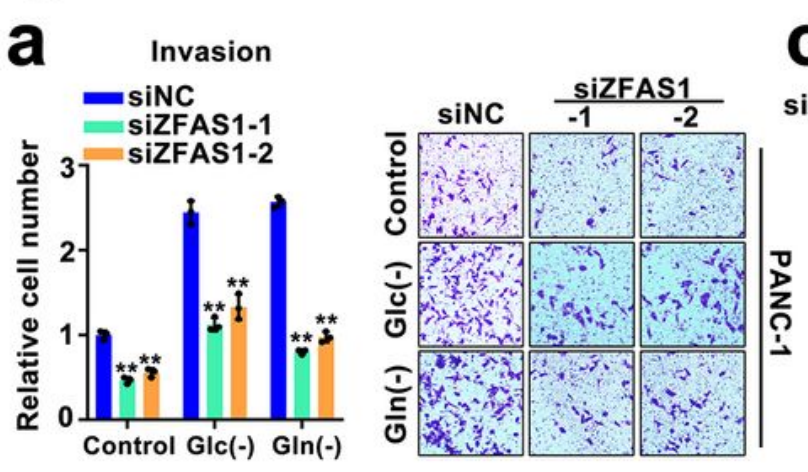

b
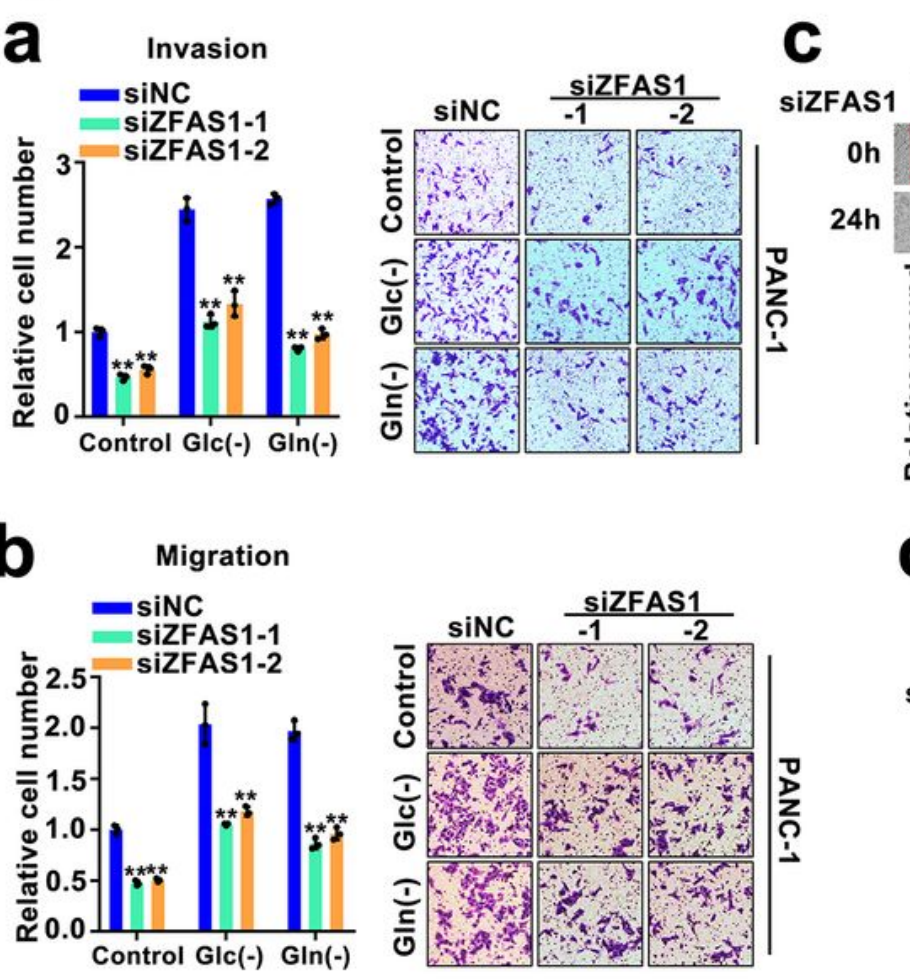

PANC-1

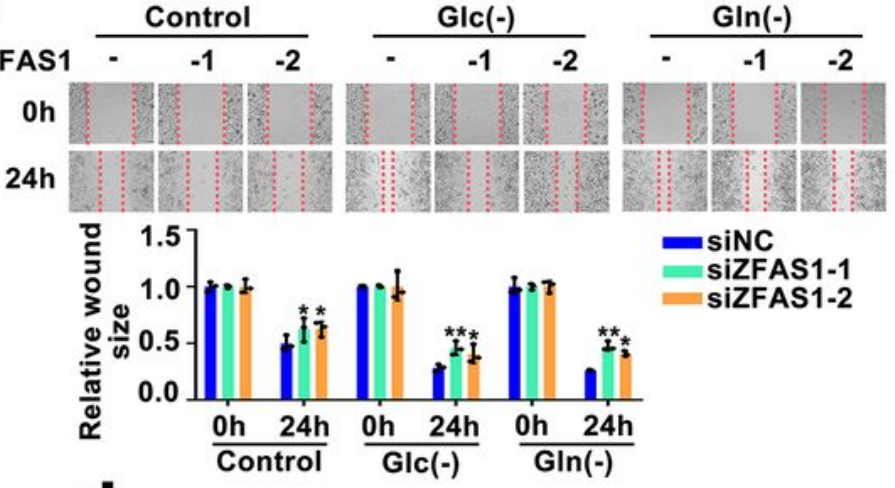

e

PANC-1
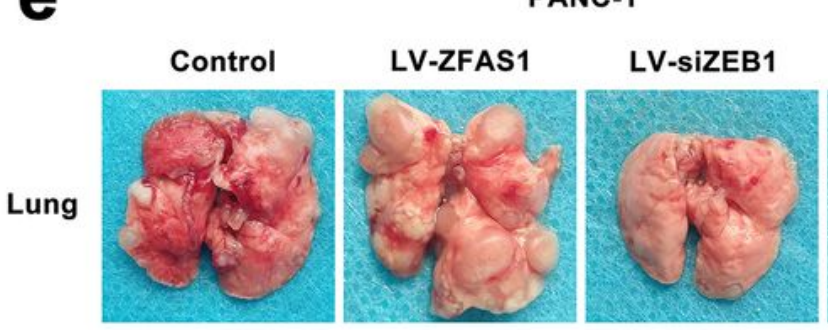

LV-ZFAS1

LV-siZEB1
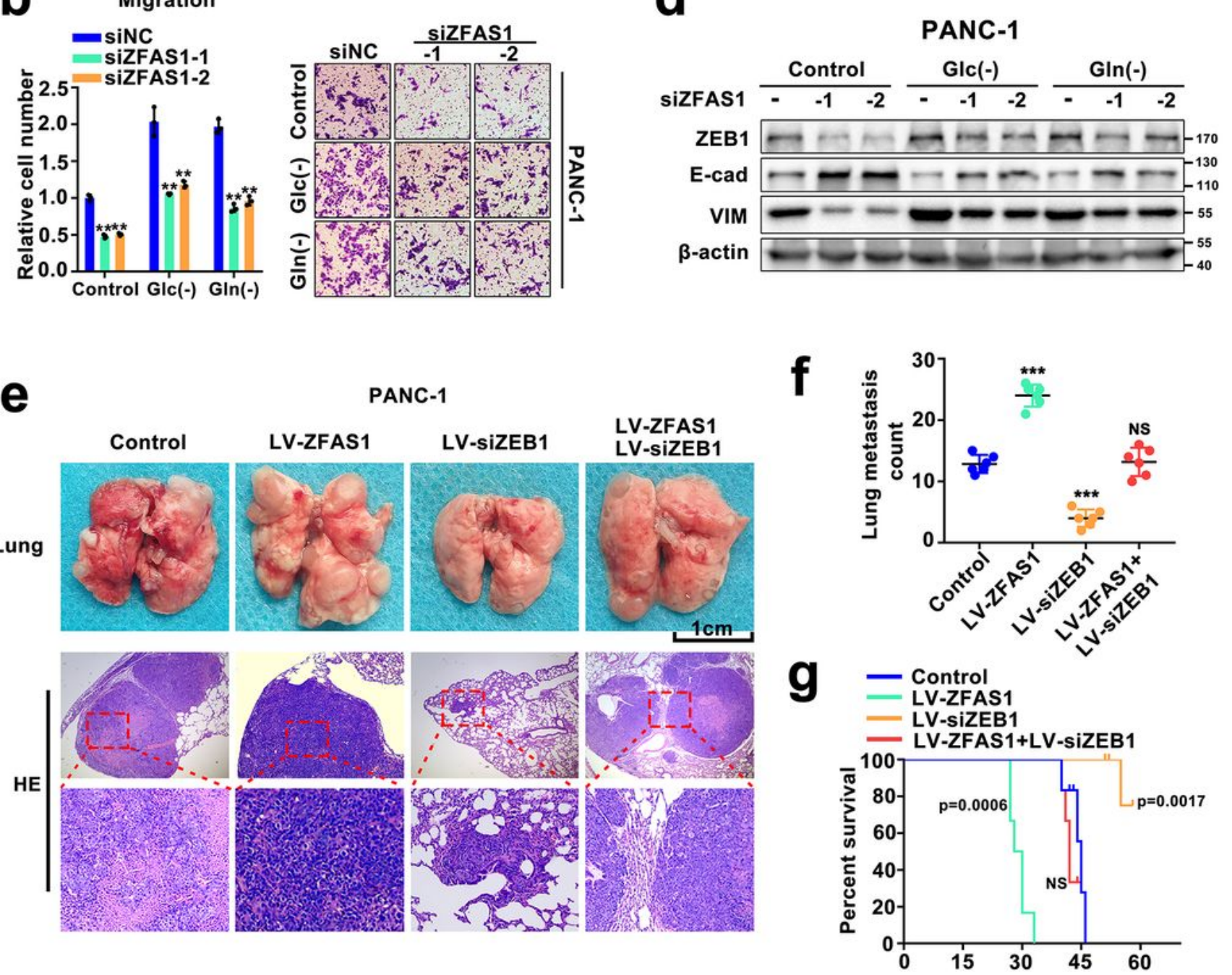

Figure 8

ZFAS1/ZEB1 feedback is critical for the nutrient stress-induced EMT and metastasis of PC cells. a Invasion of PANC-1 cells with ZFAS1 knockdown, cultured with complete medium, glucose-free medium and glutamine-free medium for $48 \mathrm{~h}$, was assessed by transwell assay. b, c Migration of PANC-1 cells with ZFAS1 knockdown, cultured with complete medium, glucose-free medium and glutamine-free medium for $48 \mathrm{~h}$, was assessed by transwell and wound healing assay. d ZEB1, E-cad and VIM protein 
levels were detected by Western Blot in ZFAS1 knockdown PANC-1 cells cultured with complete medium, glucose-free medium and glutamine-free medium. e The representative images of lungs and H\&E staining of nude mice treated with tail vein injection of PANC-1 cells stably transfected with LV-NC, LV-ZFAS1 or LV-siZEB1. $f, g$ The quantification of lung metastatic colonization ( $f$ ) and Kaplan-Meier curves $(g)$ of nude mice were displayed. Data shown represent three independent experiments. ${ }^{*} p<0.05,{ }^{\star *} p<0.01$, *** $p<0.001$. NS, no significant.

\section{Fig. 9}

a

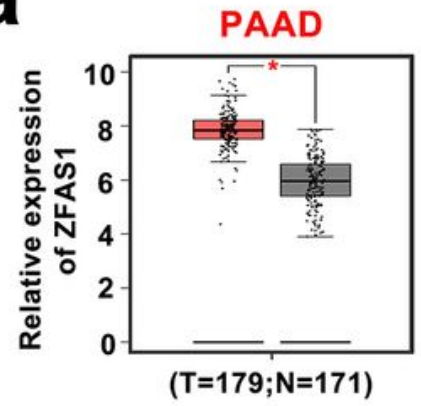

b

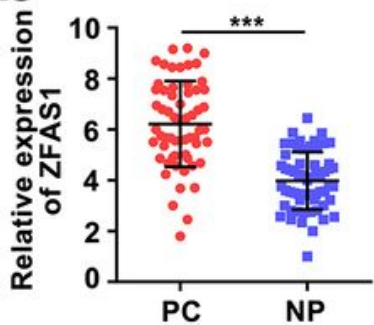

f

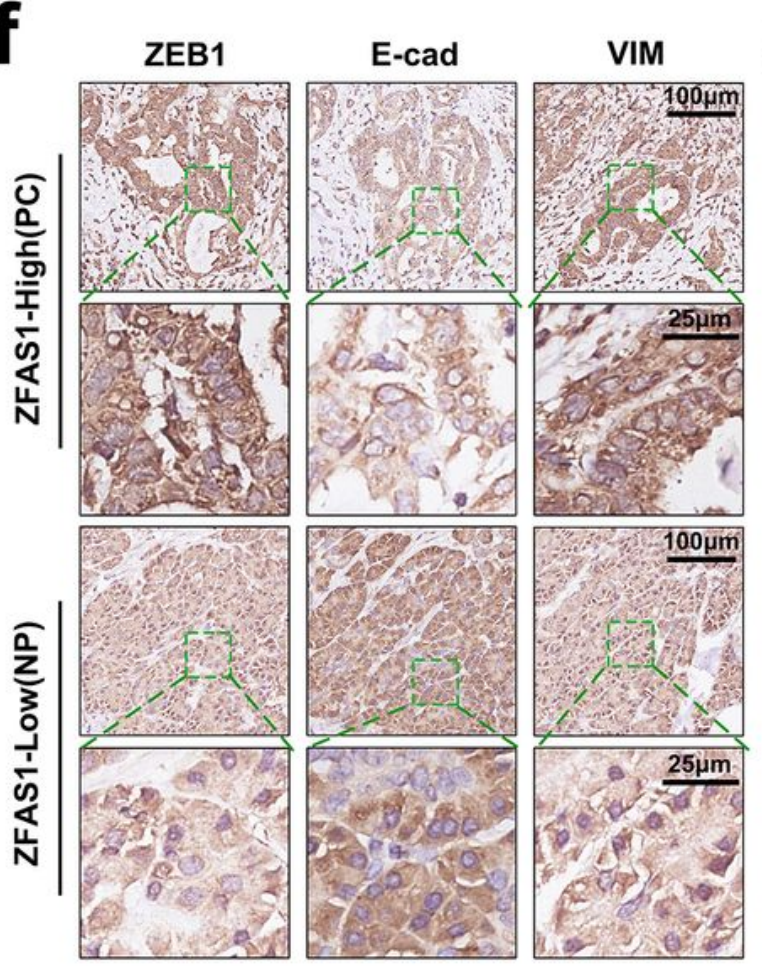

COAD

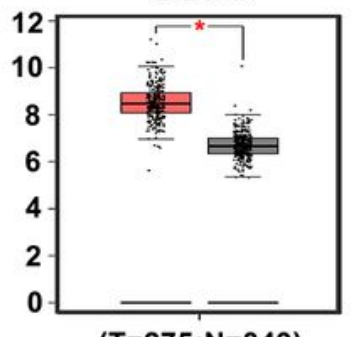

( $T=275 ; N=349$ )
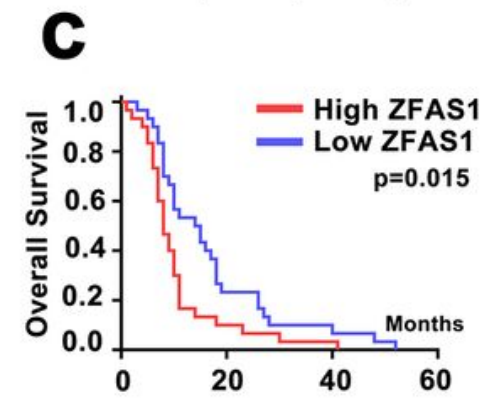

g
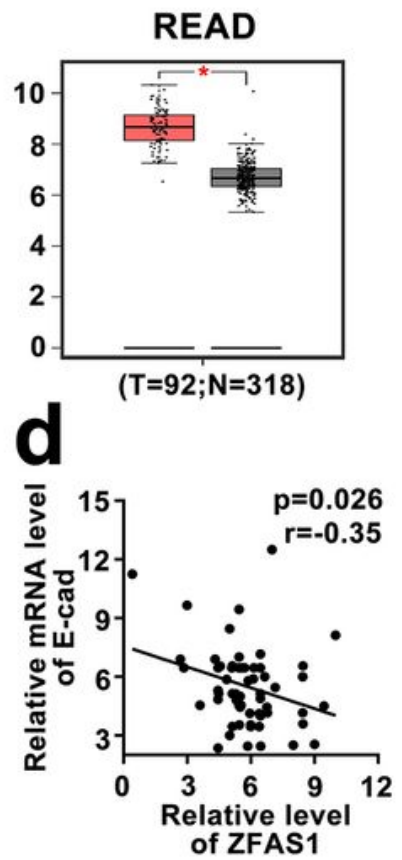

LIHC

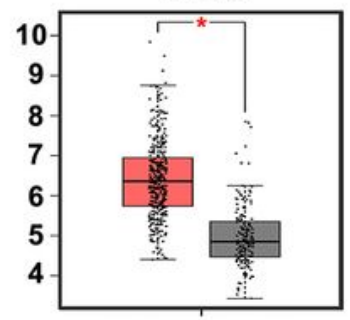

$(T=369 ; N=160$ )
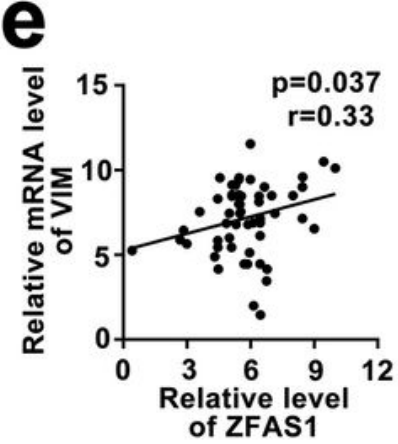

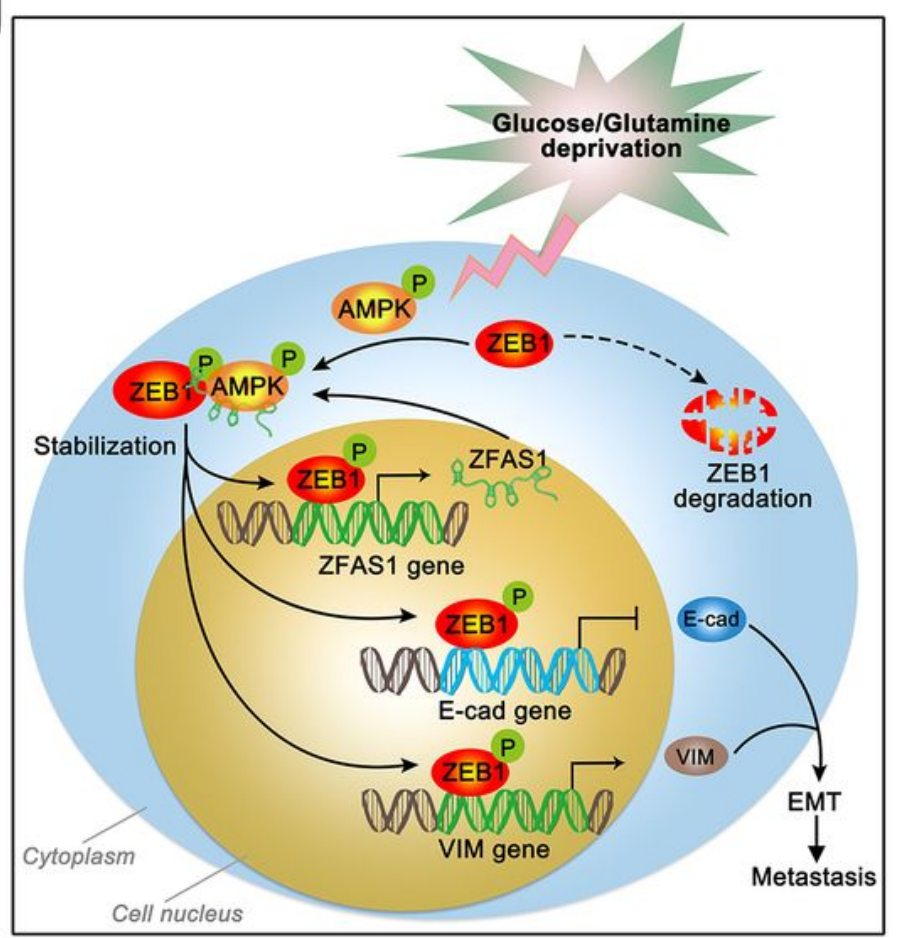

Figure 9 
ZFAS1 is highly expressed in PC and correlated with poor prognosis. a The data from GEPIA showed ZFAS1 expression in tissues of PAAD, COAD, READ and LIHC compared with normal tissues. $b$ The expression of ZFAS1 was detected by RT-qPCR in 60 PC tissues, compared with adjacent normal tissues. The Student $t$ test was used for analyzing the difference. c Overall survival of $60 \mathrm{PC}$ patients with low and high ZFAS1 was analyzed by Kaplan-Meier plots using Log-rank test. ZFAS1 expression was categorized into "high" and "low" using the median value as the cutoff point. d, e Pearson correlation analysis between ZFAS1 levels and E-cad (d), VIM (e) in PC tissues determined by RT-qPCR. $f$ IHC staining for ZEB1, E-cad and VIM in high ZFAS1 tissues (PC tissues) and low ZFAS1 tissues (adjacent normal tissues). Scale bars were shown on the right of images. $\mathrm{g}$ Graphical illustration of ZFAS1 regulation under glucose and glutamine deprivation in PC cells.

\section{Supplementary Files}

This is a list of supplementary files associated with this preprint. Click to download.

- Additionalfile1.pdf

- Additionalfile2.pdf 